\title{
PICARD SETS FOR MEROMORPHIC FUNCTIONS WITH A DEFICIENT VALUE
}

\author{
SAKARI TOPPILA
}

\section{Introduction}

Let $F$ be a family of functions meromorphic in the complex plane $C$, and $S$ a subset of $C$. We call $S$ a Picard set for $F$ if every transcendental $f \in F$ assumes every complex value with at most two exceptions infinitely often in $C-S$. We use the usual notation of the Nevanlinna theory, the Nevanlinna deficiency is defined by

$$
\delta(a, f)=\liminf _{r \rightarrow \infty} \frac{m(r, a, f)}{T(r, f)}
$$

and the Valiron deficiency by

$$
\Delta(a, f)=\limsup _{r \rightarrow \infty} \frac{m(r, a, f)}{T(r, f)} .
$$

If $F$ is the family of all functions meromorphic in the plane, the corresponding class of Picard sets is denoted by $P(M)$. Let $P(P)$ be the class of Picard sets for those meromorphic functions which have at least one Picard exceptional value. By means of a linear transformation, we see that $P(P)$ is the class of Picard sets for entire functions. The class of Picard sets for those meromorphic functions which have at least one Nevanlinna (resp. Valiron) deficient value is denoted by $P(N)$ (resp. $P(V)$ ). We see immediately that

$$
P(M) \subset P(V) \subset P(N) \subset P(P) .
$$

In this paper we shall consider the question, under which conditions a set $S$ belongs to the classes $P(N)$ or $P(V)$. First we shall consider countable sets and then the case when $S$ is a countable union of open discs. 
We shall prove

\section{Countable sets of the class $P(N)$}

Theorem 1. Let $E=\left\{a_{n}\right\}$ be a countable set whose points converge to infinity. If there exists $\varepsilon>0$ such that

$$
\left\{z: 0<\left|z-a_{n}\right|<\frac{\varepsilon\left|a_{n}\right|}{\log \left|a_{n}\right|}\right\} \cap E=\emptyset
$$

for all large $n$, then $E \in P(N)$.

This theorem is best possible in the sense that, corresponding to each realvalued function $\varphi(r)$ with $\lim _{r \rightarrow \infty} \varphi(r)=\infty$, there exists $E=\left\{a_{n}\right\}$ satisfying

$$
\left\{z: 0<\left|z-a_{n}\right|<\frac{\left|a_{n}\right|}{\varphi\left(\left|a_{n}\right|\right) \log \left|a_{n}\right|}\right\} \cap E=\emptyset
$$

for all large $n$ such that $E \notin P(P) \supset P(N)$. The existence of such a set $E$ is proved in $[11$, pp. 7-8]. Since the condition (A) is the best possible one of this type for $P(P)$, too, there arises the question whether $P(P)=P(N)$. The answer to this question is negative.

Theorem 2. There exists a countable set $E=\left\{a_{n}\right\}$ with $\lim a_{n}=\infty$ such that $E \in P(P)-P(N)$.

The following theorem shows that the condition (A) is not optimal for linear sets.

Theorem 3. Let $E=\left\{a_{n}\right\}$ be a sequence of points lying on the positive real axis and let $a_{n} \rightarrow \infty$ as $n \rightarrow \infty$. If there exists $\varepsilon>0$ such that

$$
a_{n+1}>a_{n}\left(1+\frac{\varepsilon}{\left(\log a_{n}\right)^{2}}\right)
$$

for all large $n$, then $E \in P(N)$.

The condition (B) here is optimal, even for $P(P)$, for it is proved in [12] that if $\varphi(r)$ is an increasing function such that $\varphi(r) \rightarrow \infty$ as $r \rightarrow \infty$, there exists a set $E=\left\{a_{n}\right\}$ with $\lim a_{n}=\infty$ lying on the positive real axis such that $E \notin P(P)$ and

$$
a_{n+1}>a_{n}\left(1+\frac{1}{\varphi\left(a_{n}\right)\left(\log a_{n}\right)^{2}}\right)
$$

for all large $n$. 


\section{Results for the class $P(V)$}

Corresponding to Theorem 1, we shall prove the following result for $P(V)$.

Theorem 4. If there exists $\varepsilon>0$ such that the set $E=\left\{a_{n}\right\}$ with $\lim a_{n}=\infty$ satisfies

(C)

$$
\left\{z: 0<\left|z-a_{n}\right|<\varepsilon\left|a_{n}\right|\right\} \cap E=\emptyset
$$

for all large $n$, then $E \in P(V)$.

This result is optimal, even for linear sets, for we shall prove

Theorem 5. Given any increasing function $\varphi(r)$ such that $\varphi(r) \rightarrow \infty$ as $r \rightarrow \infty$, there exists a set $E=\left\{a_{n}\right\}$ lying on the positive real axis such that $a_{n} \rightarrow \infty$ as $n \rightarrow \infty$, $E \notin P(V)$ and

$$
a_{n+1}>a_{n}\left(1+\frac{1}{\varphi\left(a_{n}\right)}\right)
$$

for all large $n$.

If in Theorem $5 \varphi(r) \rightarrow \infty$ sufficiently slowly as $r \rightarrow \infty$, then the corresponding set $E$ belongs to $P(N)$. Therefore $P(V) \neq P(N)$. Between these classes there is even a more essential difference. We denote by $U(a, r)$ the open $\operatorname{disc}|z-a|<r$. Theorem 10 proves that there exists a denumerable collection of open discs $U\left(a_{n}, d_{n}\right)$ with $\lim \left|a_{n}\right|=\infty$ such that the union of these discs belongs to $P(N)$. The class $P(V)$ does not have this property. We prove

Theorem 6. If $U\left(a_{n}, d_{n}\right)$ is any sequence of open discs such that $\lim \left|a_{n}\right|=\infty$, then the set

does not belong to $P(V)$.

$$
\bigcup_{n=1}^{\infty} U\left(a_{n}, d_{n}\right)
$$

\section{Comparison of $P(V)$ and $P(M)$}

As in Theorems 1 and 4, it is proved in [10] that if the set $E=\left\{a_{n}\right\}$ satisfies

$$
\left|a_{n+1}\right|>\varepsilon\left|a_{n}\right|^{2}
$$

for some $\varepsilon>0$ and for all large $n$, then $E \in P(M)$, and in [12] it is proved that if $\varphi(r) \rightarrow \infty$ as $r \rightarrow \infty$, there exists $E=\left\{a_{n}\right\}$ lying on the positive real axis such that $E \notin P(M)$ and

$$
a_{n+1}>\frac{a_{n}^{2}}{\varphi\left(a_{n}\right)}
$$

for all large $n$. We conclude that $P(V) \neq P(M)$. The conditions (C) and (1) are quite far from each other and therefore we try to characterize those functions 
which make the difference between $P(V)$ and $P(M)$ so large. We denote by $\Sigma$ the extended complex plane and prove

Theorem 7. If $f$ is a transcendental meromorphic function such that the set $E=\left\{a_{n}\right\}=f^{-1}(\{0,1, \infty\})$ satisfies the condition

$$
\lim _{n \rightarrow \infty}\left|a_{n+1} / a_{n}\right|=\infty,
$$

then

$$
\limsup _{r \rightarrow \infty}\left(\sup _{w \in \Sigma} n(r, w)-\inf _{w \in \Sigma} n(r, w)\right) \leqq 2,
$$

and for any two complex values $a$ and $b, \lim \sup _{r \rightarrow \infty}|n(r, a)-n(r, b)| \leqq 1$.

Furthermore, we shall show that a meromorphic function may have at most three so thinly distributed values that $(E)$ is satisfied.

Theorem 8. If $f$ is a transcendental meromorphic function in the plane and $w_{4}$ is different from 0,1 and $\infty$, then the set $E=\left\{a_{n}\right\}=f^{-1}\left(\left\{0,1, w_{4}, \infty\right\}\right)$ satisfies

$$
\liminf _{n \rightarrow \infty}\left|\frac{a_{n+1}}{a_{n}}\right|<\infty .
$$

In the other direction, we shall prove

Theorem 9. For any $M>1$ there exists a transcendental meromorphic function $f$ such that the set

$$
E=\left\{a_{n}\right\}=f^{-1}(\{0,1, M, \infty\})
$$

satisfies

$$
\liminf _{n \rightarrow \infty}\left|\frac{a_{n+1}}{a_{n}}\right|=M .
$$

\section{Further results for the class $P(N)$}

From the results of Anderson and Clunie [1] it follows that if $q>1$, the set $E=\left\{a_{n}\right\}$ satisfies

$$
\left|a_{n+1} / a_{n}\right| \geqq q
$$

for all $n$, and the radii $d_{n}$ are chosen such that

$$
\left(\log \left|a_{n}\right|\right)^{2}=o\left(\log \frac{1}{d_{n}}\right),
$$

then the union of the discs $U\left(a_{n}, d_{n}\right)$ belongs to $P(N)$. It is proved in [15] that the condition (b) here can be replaced by

$$
\log \frac{1}{d_{n}} \geqq K\left(\log \left|a_{n}\right|\right)^{2},
$$


where $K>0$ depends only on $q$, and still $\cup U\left(a_{n}, d_{n}\right) \in P(N)$, and in the other direction, if $K$ in $\left(b^{\prime}\right)$ is taken too small,

$$
K=\frac{1}{2 \log q}
$$

there exist $U\left(a_{n}, d_{n}\right)$ satisfying (a) and $\left(\mathrm{b}^{\prime}\right)$ such that $\cup U\left(a_{n}, d_{n}\right)$ does not belong to $P(P)$. We shall relieve (a) and prove

Theorem 10. Let $E=\left\{a_{n}\right\}$ be a complex sequence such that $\lim a_{n}=\infty$, $\left|a_{n}\right|>e$, and

$$
\left\{z: 0<\left|z-a_{n}\right|<\frac{\left|a_{n}\right|}{\left(\log \left|a_{n}\right|\right)^{\alpha}}\right\} \cap E=\emptyset
$$

for some $\alpha, 0<\alpha<1$, and for all $n$. If the radii $d_{n}$ are chosen by the equation

$$
\log \frac{1}{d_{n}}=\left(\log \left|a_{n}\right|\right)^{2+\beta},
$$

where $\beta>2 \alpha$, then the set

$$
S=\bigcup_{n=1}^{\infty} U\left(a_{n}, d_{n}\right)
$$

belongs to the class $P(N)$.

Here $\beta$ cannot be smaller than $2 \alpha$, for it is proved in [13] that if $\beta<2 \alpha$, there exists $S=\cup U\left(a_{n}, d_{n}\right)$ satisfying $(\mathrm{H})$ and $(\mathrm{I})$, and not belonging to $P(P)$.

Theorem 3 follows as a special case from the following

Theorem 11. Let $E=\left\{a_{n}\right\}$ lie on the positive real axis, $e<a_{1}<a_{2}<\ldots, a_{n} \rightarrow \infty$ as $n \rightarrow \infty, \varepsilon>0$, and

$$
a_{n+1}>a_{n}\left(1+\frac{\varepsilon}{\left(\log a_{n}\right)^{\alpha}}\right)
$$

for some $\alpha, 0<\alpha \leqq 2$, and for all $n$. If the radii $d_{n}$ are chosen by the equation

$$
\log \frac{1}{d_{n}}=H\left(\log a_{n}\right)^{2+\alpha}
$$

where $H=4800\left(1+\varepsilon^{-2}\right)(100)^{2+\alpha}$, then the union of the discs $U\left(a_{n}, d_{n}\right)$ belongs to $P(N)$.

In the other direction, it is proved in [14] that if $\varepsilon=1 / 7, H=1 / 8$ and $0<\alpha \leqq 2$, then $E$ and $d_{n}$ satisfying $(\mathrm{J})$ and $(\mathrm{K})$ can be chosen such that the intersection of the positive real axis and the union of the discs $U\left(a_{n}, d_{n}\right)$ does not belong to $P(P)$. 


\section{Some results needed in the proofs}

We denote $U(\infty, \delta)=\{z:|z|>1 / \delta\}$. We shall need the following

Lemma 1. There exist positive constants $M_{1}$ and $M_{2}$ depending only on $w_{3}$ such that if $f$ is meromorphic in an annulus $r<|z|<R$ and omits there three different values 0,1 and $w_{3}$, then, if $R>M_{1} r$, the image of $|z|=\sqrt{r R}$ under $f$ is contained in

$$
U\left(a, M_{2}(\log (R / r))^{-1 / 4}\right)
$$

for some finite or infinite complex $a$.

Proof. Let $f$ be meromorphic and omit the values 0,1 and $w_{3}$ in $r<|z|<R$, where $\log (R / r)>8 \pi$. We denote $z_{0}=\sqrt{R r}=\exp \left(\zeta_{0}\right)$. We choose $g$ to be one of the functions $1 / f$ and $1 /(f-1)$ such that $\left|g\left(z_{0}\right)\right| \leqq 2$. The function $g\left(e^{\zeta}\right)$ is regular in $U\left(\zeta_{0},(1 / 2) \log (R / r)\right)$ and omits there two finite values. Therefore it follows from Schottky's theorem that there exists $M_{3}>0$ depending only on $w_{3}$ such that $\left|g\left(e^{\zeta}\right)\right| \leqq M_{3}$ in $\left|\zeta-\zeta_{0}\right| \leqq(1 / 4) \log (R / r)$. The function

$$
h(\zeta)=\frac{g\left(e^{\zeta}\right)-g\left(e^{\zeta_{0}}\right)}{\zeta-\zeta_{0}}
$$

is regular in $U\left(\zeta_{0},(1 / 4) \log (R / r)\right)$, and on the boundary of this disc $h$ satisfies

$$
|h(\zeta)| \leqq \frac{8 M_{3}}{\log (R / r)} .
$$

It follows from the maximum principle that (i) holds on the segment $\zeta=\zeta_{0}+i \varphi$, $-\pi \leqq \varphi \leqq \pi$, and we get

$$
\left|g(z)-g\left(z_{0}\right)\right| \leqq \frac{8 \pi M_{3}}{\log (R / r)}
$$

on $|z|=\sqrt{r \bar{R}}$. Lemma 1 follows from (ii) by an easy computation.

Lemma 2. Let $f$ be meromorphic in the plane and

$$
E=f^{-1}\left(\left\{0,1, w_{3}\right\}\right),
$$

where $w_{3}$ is different from 0 and 1 . For any $M>0$, there exists a constant $K=K\left(M, w_{3}\right)$ such that if $|f(b)| \geqq 2 M$ and $|f(\zeta)| \leqq M$, then the disc

$$
U(\zeta, K|b-\zeta|)
$$

contains at least two points of $E$.

Proof. Let $M_{1}$ and $M_{2}$ be as in Lemma 1 . We choose $K>M_{1}^{2}$ so large that if $a$ is any complex point, the set $U(a, 2 d)$, where $d=M_{2}((1 / 2) \log K)^{-1 / 4}$, contains at most one of the values $f(b)$ and $f(\zeta)$, and at most one of the points 0,1 and $w_{3}$. Let us suppose that $U(\zeta, K|b-\zeta|)$ contains at most one point of $E$. If $U(\zeta,|b-\zeta| \sqrt{K}) \cap E$ 
$=\emptyset$, we set $r=|b-\zeta|$, and otherwise we set $r=|b-\zeta| \sqrt{K}$. Then $f$ omits the values 0,1 and $w_{3}$ in the annulus $r<|z-\zeta|<r \sqrt{K}$, and it follows from Lemma 1 that the image of the circle $\gamma:|z-\zeta|=K^{1 / 4} r$ is contained in $U(a, d)$ for some complex $a$. Let $D$ be the open disc bounded by $\gamma$. Since the image of the boundary of $D$ is contained in $U(a, d)$ and at least one of the values $f(b)$ and $f(\zeta)$ lies outside $U(a, d), f$ takes in $D$ all values lying outside $U(a, d)$. This implies that $f$ takes in $D$ at least two of the values 0,1 and $w_{3}$, and we see that $D \subset U(\zeta, K|\zeta-b|)$ contains at least two points of $E$. So we have proved that the assumption that $U(\zeta, K|b-\zeta|)$ contains at most one point of $E$, leads to a contradiction. Lemma 2 is proved.

Let $f$ be meromorphic in the plane and let $w_{1}, w_{2}$ and $w_{3}$ be three different complex values. Let $a_{n}$ be the sequence of the distinct roots of the equations $f(z)=w_{1}$, $f(z)=w_{2}$ and $f(z)=w_{3}$. We denote by $n(r)$ the number of the $a_{n}$ lying in $|z| \leqq r$, and

$$
N(r)=\int_{0}^{r} \frac{n(t)-n(0)}{t} d t+n(0) \log r .
$$

From Theorem 2.5 of Hayman [5, p. 47] we get the following

Lemma 3. Let $f$ and $N(r)$ be as above. Then

$$
T(r, f) \leqq(1+o(1)) N(r)
$$

as $r \rightarrow \infty$ outside a set $B$ of finite linear measure.

Lemma 4. Let $f$ be transcendental and meromorphic in the plane such that $\delta(\infty, f)>0$ and

$$
T(r, f)=O\left((\log r)^{M}\right)
$$

for some finite $M$. If there exists $\alpha>0$ such that

satisfies

$$
E=\left\{a_{n}\right\}=f^{-1}(\{0,1\})
$$

$$
\left\{z: 0<\left|z-a_{n}\right|<\frac{\left|a_{n}\right|}{\left(\log \left|a_{n}\right|\right)^{\alpha}}\right\} \cap E=\emptyset
$$

for all large $n$, then there exists a real increasing sequence $\sigma_{n}$ such that $\sigma_{n} \rightarrow \infty$ as $n \rightarrow \infty$,

$$
\min \left\{|f(z)|:|z|=\sigma_{n}\right\}=1
$$

$|f(z)|>1$ in $\sqrt{\sigma_{n}}<|z|<\sigma_{n}$ and

$$
\log |f(z)| \geqq\left(\frac{1}{3}+o(1)\right) \delta(\infty, f) T(|z|, f)
$$

for all $z$ lying in $\sqrt{\sigma_{n}} \leqq|z| \leqq \sigma_{n} / 2$. 
Proof. It follows from (2) that we may choose $\beta, 3 / 2 \leqq \beta \leqq M$, such that

$$
T(r, f)=O\left((\log r)^{\beta}\right)
$$

and

$$
T(r, f) \neq O\left((\log r)^{\beta-1 / 2}\right) .
$$

Since

$$
n(r, a) \log r \leqq \int_{r}^{r^{2}} \frac{n(t, a)}{t} d t=N\left(r^{2}, a\right)-N(r, a),
$$

we deduce from (i) that

$$
n(r, a)=O\left((\log r)^{\beta-1}\right)
$$

for any complex $a$, and from (ii) it follows that there exists a real sequence $R_{n}$ with $\lim R_{n}=\infty$ such that

$$
T\left(R_{n}, f\right)>\left(\log R_{n}\right)^{\beta-1 / 2}
$$

for all $n$.

Let $b$ be a complex value such that $|b|<1$ and

$$
N(r, b)=(1+o(1)) T(r, f) .
$$

Let $b_{n}$ be the sequence of the $b$-points of $f$ and

$$
B=\bigcup_{\left|b_{k}\right|>e} U\left(b_{k},\left|b_{k}\right|\left(\log \left|b_{k}\right|\right)^{-2(\alpha+\beta)}\right) .
$$

We denote $d(z)=\min \left\{\left|z-b_{k}\right|: k=1,2, \ldots\right\}$. Using the Poisson-Jensen formula, we get for all $z=r e^{i \varphi}$,

$$
\begin{aligned}
& \log |f(z)-b| \geqq \frac{1}{2 \pi} \int_{0}^{2 \pi} \log \left|f\left(2 r e^{i \theta}\right)-b\right| \frac{(2 r)^{2}-r^{2}}{(2 r)^{2}-4 r^{2} \cos (\theta-\varphi)+r^{2}} d \theta \\
&-\sum_{\left|b_{k}\right| \geqq 2 r} \log \left|\frac{(2 r)^{2}-\bar{b}_{k} z}{2 r\left(z-b_{k}\right)}\right| .
\end{aligned}
$$

This implies, together with the fact that $m(t, b)=o(T(t, f))$, that

$$
\log |f(z)| \geqq\left(\frac{1}{3}+o(1)\right) \delta(\infty, f) T(2 r, f)-n(2 r, b) \log \frac{4 r}{d(z)} .
$$

Let $z=r e^{i \varphi}$ lie in $R_{n}<|z|<R_{n}^{3}$ outside $B$. Then

$$
d(z) \geqq r(2 \log r)^{-2(\alpha+\beta)},
$$

and we see from (iii) and (iv) that

$$
n(2 r, b) \log (4 r / d(z))=O\left(\left(\log R_{n}\right)^{\beta-1} \log \log R_{n}\right)=o\left(T\left(R_{n}, f\right)\right) .
$$

Therefore it follows from (vi) that $f$ satisfies (4) in $R_{n}<|z|<R_{n}^{3}$ outside the set $B$. Let us suppose that there exists some $b_{k}$ lying in $2 R_{n}<|z|<R_{n}^{3} / 2$. The sum of the 
radii of those discs of the set $B$ which meet the annulus $\left|b_{k}\right| / 2<|z|<2\left|b_{k}\right|$ is at most

$$
2 n\left(R_{n}^{3}, b\right)\left|b_{k}\right|\left(\log \left|b_{k}\right|\right)^{-2(\alpha+\beta)}=o\left(\left|b_{k}\right|\left(\log \left|b_{k}\right|\right)^{-2 \alpha}\right),
$$

and we see that there exists $d_{k}$,

$$
0<d_{k}<\left|b_{k}\right|\left(\log \left|b_{k}\right|\right)^{-2 \alpha},
$$

such that the circle $\left|z-b_{k}\right|=d_{k}$ does not meet $B$. It follows from (3) and (vii) that $f$ takes at most one of the values 0 and 1 in $U\left(b_{k}, d_{k}\right)$, and since (4) is true on the boundary of this disc, we conclude from the minimum principle that

$$
\log |b|=\log \left|f\left(b_{k}\right)\right| \geqq\left(\frac{1}{3}+o(1)\right) \delta(\infty, f) T\left(\left|b_{k}\right|, f\right) .
$$

This is not possible if $\left|b_{k}\right|$ is large, and we deduce that

for all large $n$.

$$
B \cap\left\{z: 4 R_{n}<|z|<R_{n}^{3} / 4\right\}=\emptyset
$$

For large values of $n$, we may choose $\sigma_{n}>R_{n}^{3} / 4$ such that $|f(z)|>1$ in $4 R_{n}<$ $|z|<\sigma_{n}$ and

$$
\min \left\{|f(z)|:|z|=\sigma_{n}\right\}=1
$$

Let $\zeta=r e^{i \varphi}$ lie in $\sqrt{\sigma_{n}} \leqq|z| \leqq \sigma_{n} / 2$. Since $f$ has no $b$-points in $4 R_{n}<|z| \leqq \sigma_{n}$, we conclude that $n(2 r, b)=n\left(4 R_{n}, b\right), d(\zeta) \geqq r / 2$ and

$$
n(2 r, b) \log (4 r / d(\zeta))=O\left(\left(\log R_{n}\right)^{\beta-1} \log 8\right)=o\left(T\left(R_{n}, f\right)\right)
$$

Now we see from (vi) that $f$ satisfies (4) in $\sqrt{\sigma_{n}} \leqq|z| \leqq \sigma_{n} / 2$, and Lemma 4 is proved.

Following Hayman [6], we shall call an $\varepsilon$-set any countable set of circles not containing the origin, and subtending angles at the origin whose sum is finite. Hayman [6] has proved the following

Theorem A. If an integral function $f$ satisfies $\log M(r, f)=O\left((\log r)^{2}\right)$, then

$$
\log |f(z)|=(1+o(1)) \log M(r, f)
$$

as $z=r e^{i \varphi} \rightarrow \infty$ outside an $\varepsilon$-set.

Valiron [16] has proved the following

Theorem B. If a meromorphic function $f$ satisfies $T(r, f)=O\left((\log r)^{2}\right)$, then

$$
T(r, f)=(1+o(1)) \max \{N(r, a), N(r, b)\}
$$

for any two complex values $a$ and $b$. 


\section{Proof of Theorem 1}

Contrary to the assertion of Theorem 1, let us suppose that there exists a transcendental meromorphic function $f$ with a Nevanlinna deficient value $w$ such that the set

$$
E=\left\{a_{n}\right\}=f^{-1}\left(\left\{w_{1}, w_{2}, w_{3}\right\}\right)
$$

satisfies (A) for some $\varepsilon>0$ and for some choice of the three different values $w_{1}, w_{2}$ and $w_{3}$. We may suppose, without loss of generality, that $w=\infty, w_{1}=0$ and $w_{2}=1$, $w_{3}$ being an infinite or finite complex value, different from 0 and 1 .

Let $n(r)$ be the counting function of $E$. It follows from (A) that

and we conclude that

$$
n\left(e^{s}\right)-n\left(e^{s-1}\right)=O\left(s^{2}\right),
$$

$$
n\left(e^{s}\right)=O(1)+\sum_{k=1}^{s}\left(n\left(e^{k}\right)-n\left(e^{k-1}\right)\right)=O\left(\sum_{k=1}^{s} k^{2}\right)=O\left(s^{3}\right) .
$$

This implies that $n(r)=O\left((\log r)^{3}\right)$, and therefore the integrated counting function of $E$ satisfies $N(r)=O\left((\log r)^{4}\right)$. It follows from Lemma 3 that $T(r, f) \leqq$ $(1+o(1)) N(2 r)$ for all large values of $r$, and we deduce that $f$ satisfies $T(r, f)=$ $O\left((\log r)^{4}\right)$. This implies that we may apply Lemma 4 .

Let the sequence $\sigma_{n}$ be as in Lemma 4 . We choose $b_{n}$ lying on the circle $|z|=\sigma_{n}$ such that $\left|f\left(b_{n}\right)\right|=1$. Since $f$ is transcendental, we conclude that

$$
\liminf _{r \rightarrow \infty} \frac{T(r, f)}{\log r}=\infty .
$$

Therefore we deduce from (4) that there exists a sequence $K_{n}$ with $\lim K_{n}=\infty$ such that

$$
\log |f(z)| \geqq K_{n}^{2} \log \sigma_{n}
$$

on $|z|=\sigma_{n} / e$. The function $\omega(z)=\log \left(\sigma_{n} /|z|\right)$ is harmonic in the annulus $\sigma_{n} / e \leqq$ $|z| \leqq \sigma_{n}$, and on the boundary of this annulus we have

$$
\log |f(z)| \geqq K_{n}^{2} \omega(z) \log \sigma_{n} .
$$

Since $\log |f(z)|$ is superharmonic in $\sigma_{n} / e \leqq|z| \leqq \sigma_{n}$, it follows from the minimum principle that (ii) holds in this annulus. We set

$$
z_{n}=b_{n}\left(1-\frac{1}{K_{n} \log \sigma_{n}}\right) .
$$

Then it follows from (ii) that $\log \left|f\left(z_{n}\right)\right| \geqq K_{n}$, and we see from Lemma 2 that 
the disc

$$
C_{n}=U\left(b_{n}, \frac{K\left|b_{n}\right|}{K_{n} \log \left|b_{n}\right|}\right)
$$

contains at least two points of $E$. However, since $\lim K_{n}=\infty$, it follows from (A) that if $n$ is large, then $C_{n}$ contains at most one point of $E$. We are led to a contradiction and Theorem 1 is proved.

\section{Proof of Theorem 2}

Let $r_{1}=e^{10}$ and $r_{n-1}=\log \log \log r_{n}$ for $n \geqq 2$. We set

$$
f(z)=z \prod_{n=1}^{\infty}\left(\left(1-\frac{z}{r_{n}}\right)^{2}\left(1-\frac{z}{r_{n}-\sqrt{r_{n}}}\right)^{-1}\right) .
$$

Then $n(r, \infty, f)=(1 / 2+o(1)) n(r, 0, f)$, and we see that $\delta(\infty, f) \geqq 1 / 2$. This implies that the set $E=\left\{a_{n}\right\}=f^{-1}(\{0,1, \infty\})$ does not belong to the class $P(N)$. We assume that the sequence $a_{n}$ is arranged in the order of increasing moduli. We see by an easy computation that if $k$ is large, then $a_{4 k-1}=r_{k}-\sqrt{r_{k}}$ and $a_{4 k+p} \in U\left(r_{k}, r_{k}^{-2}\right)$ for $p=0,1,2$. Let $f(\zeta)=1$ and $\zeta \in U\left(r_{k}, r_{k}^{-2}\right)$. Then

$$
(-1)^{k}+o(1)=r_{k}^{k-2} \sqrt{r_{k}}\left(\zeta-r_{k}\right)^{2} \prod_{t=1}^{k-1}\left(r_{t}^{-2}\left(r_{t}-\sqrt{r_{t}}\right)\right)
$$

and we conclude that

$$
\log |\zeta-z|^{-1}=\frac{1}{2}\left(k-2+\frac{1}{2}\right) \log r_{k}+o\left(\log \log r_{k}\right)
$$

for any choice $\zeta \neq z,\{\zeta, z\} \subset\left\{a_{4 k}, a_{4 k+1}, a_{4 k+2}\right\}$.

Let us suppose now that $E \notin P(P)$. Then there exists a trancendental entire function $g$ such that

$$
E(g)=g^{-1}(\{0,1\}) \subset E \cup U\left(0, r_{0}\right)
$$

for some $r_{0}>0$. Since $M(r, g) \rightarrow \infty$ as $r \rightarrow \infty$, we may conclude from Schottky's theorem that $|g(z)| \geqq 4$ on the circles $\gamma_{k}:|z|=r_{k} / 2$ and $\Gamma_{k}:|z|=2 r_{k}$ for all large $k$. We denote by $D_{k}$ the annulus which is bounded by $\gamma_{k}$ and $\Gamma_{k}$.

Let us suppose that $a_{n} \in D_{k}$ is a multiple root of the equation $g(z)=0$ with multiplicity $m \geqq 4$. Since $|g(z)| \geqq 4$ on the boundary of $D_{k}$ and $D_{k}$ contains only four points of $E$, there exists a region $G \subset C$ such that the image of the boundary of $G$ is contained in the segment $w=u+i v: 0 \leqq u \leqq 1, v=0$. This implies together with the maximum principle that $\operatorname{Im} g(z) \equiv 0$ on $G$, and therefore $g(z) \equiv$ constant on $G$. This is a contradiction, and we conclude that the equation $g(z)=0$ may have only a finite number of roots with multiplicity $m \geqq 4$. It follows from Lemma 3 
that $T(r, g)=O\left((\log r)^{2}\right)$, and we may write

$$
g(z)=P(z) \prod_{n=1}^{\infty}\left(1-z / a_{n}\right)^{s_{n}}
$$

where $P$ is a polynomial and $s_{n} \in\{0,1,2,3\}$ for any $n$.

If $k$ is large and $z$ is a boundary point of the disc $U\left(r_{k}-\sqrt{r_{k}},(1 / 2) \sqrt{r_{k}}\right)$, then

$$
\log |g(z)| \geqq 100 \log |z|+\left(n\left(2 r_{k}, 0, g\right)-n\left(r_{k} / 2,0, g\right)\right) \log \left(8 r_{k}^{-1 / 2}\right),
$$

and since $n\left(2 r_{k}, 0, g\right)-n\left(r_{k} / 2,0, g\right) \leqq 12$, we conclude that $|g(z)| \geqq 4$. Since $g$ omits at least one of the values 0 and 1 in $U\left(r_{k}-\sqrt{r_{k}},(1 / 2) \sqrt{r_{k}}\right)$ and $|g(z)| \geqq 4$ on the boundary of this disc, it follows from the minimum principle that $|g(z)| \geqq 2$ in this disc. This implies that $r_{k}-\sqrt{r_{k}} \notin E(g)$, and therefore $D_{k}$ contains at most three points of $E(g)$. As before, we see now that if $n\left(2 r_{k}, 0, g\right)-n\left(r_{k} / 2,0, g\right) \geqq 3$, then there exists a region $G$ contained in the open disc bounded by $\Gamma_{k}$ such that the image of the boundary of $G$ is contained in the real axis. However, this is impossible, and we conclude that $n\left(2 r_{k}, 0, g\right)-n\left(r_{k} / 2,0, g\right) \leqq 2$ for all large $k$. We denote by $p_{k}$ the number of the roots of the equation $g(z)=0$ in $D_{k}$ when the multiple roots are counted according to multiplicity. Then $p_{k} \leqq 2$ for all large $k$, and it follows from Rouche's that the equation $g(z)=1$ has $p_{k}$ roots in $D_{k}$, too.

Let $k$ be large and $p_{k}>0$. If $p_{k}=2$, then one of the functions $g$ and $1-g$ has a double zero at one of the points $a_{4 k}, a_{4 k+1}$ and $a_{4 k+2}$, and takes the value 1 at the two remaining points. We may suppose that in this case $g$ has this property. In both cases, $p_{k}=2$ or $p_{k}=1$, we denote by $\zeta$ the zero of $g$ lying in $D_{k}$ and let $z \in D_{k}$ be such a point that $g(z)=1$. Then $\{\zeta, z\} \subset\left\{a_{4 k}, a_{4 k+1}, a_{4 k+2}\right\}$. It follows from (ii) and the choice of the sequence $r_{n}$ that there exists a positive integer $m(k)$ such that

$$
0=\log |g(z)|=m(k) \log r_{k}+O\left(\log r_{k-1}\right)+p_{k} \log \left|\frac{\zeta-z}{r_{k}}\right| .
$$

This implies that

$$
\log |\zeta-z|^{-1}=\frac{1}{p_{k}}\left(m(k)-p_{k}\right) \log r_{k}+o\left(\log \log r_{k}\right),
$$

and comparing this with (i) we get $(1 / 4) \log r_{k}=o\left(\log \log r_{k}\right)$. This is impossible for large values of $k$, and therefore $g$ has only a finite number of zeros. This implies, together with the facts that $g$ is entire and has order zero, that $g$ is a polynomial. We are led to a contradiction, and therefore $E \in P(P)$. This completes the proof of Theorem 2 . 


\section{Proof of Theorem 4}

Contrary to the assertion of Theorem 4, let us suppose that there exists a meromorphic transcendental function $f$ with $\Delta(\infty, f)>0$ such that

$$
E=\left\{a_{n}\right\}=f^{-1}\left(\left\{0,1, w_{3}\right\}\right)
$$

satisfies (C) for some $\varepsilon>0, w_{3}$ being different from 0 and 1. It follows from (C) that the integrated counting function of $E$ satisfies $N(r)=O\left((\log r)^{2}\right)$, and we conclude from Lemma 3 that $f$ satisfies $T(r, f)=O\left((\log r)^{2}\right)$. Therefore we may write $f(z)=f_{1}(z) / f_{2}(z)$, where $f_{1}$ and $f_{1}$ are entire functions with no zeros in common and both of them satisfying $T\left(r, f_{k}\right)=O\left((\log r)^{2}\right)$. It follows from Theorem B that

and

$$
N(r, 0, f)=N\left(r, 0, f_{1}\right)=(1+o(1)) T\left(r, f_{1}\right)
$$

$$
N(r, \infty, f)=N\left(r, 0, f_{2}\right)=(1+o(1)) T\left(r, f_{2}\right),
$$

and from Theorem $\mathrm{A}$ it follows that

$$
T\left(r, f_{k}\right)=(1+o(1)) \log M\left(r, f_{k}\right) .
$$

Now we deduce from Theorem $\mathrm{A}$ that

$$
\begin{aligned}
\log |f(z)| & =\log \left|f_{1}(z)\right|-\log \left|f_{2}(z)\right| \\
& =\log M\left(r, f_{1}\right)-\log M\left(r, f_{2}\right)+o(T(r, f)) \\
& =N(r, 0, f)-N(r, \infty, f)+o(T(r, f))
\end{aligned}
$$

outside an $\varepsilon$-set.

We choose a sequence $R_{n}$ with $\lim R_{n}=\infty$ such that

$$
N\left(R_{n}, \infty, f\right)<\left(1-\frac{1}{2} \Delta(\infty, f)\right) T\left(R_{n}, f\right)
$$

for all $n$. It follows from Theorem $\mathrm{B}$ that

$$
N\left(R_{n}, 0, f\right)=(1+o(1)) T\left(R_{n}, f\right) .
$$

For large values of $n$, we may choose $r_{n}$ such that $R_{n} / 2<r_{n} \leqq R_{n}$ and that the circle $|z|=r_{n}$ lies outside the $\varepsilon$-set which is the exceptional set for the formula (i). Since $T(r, f)=O\left((\log r)^{2}\right)$, we conclude that $n(r, 0, f)=O(\log r)$ and

$$
\begin{aligned}
N\left(r_{n}, 0, f\right) & =N\left(R_{n}, 0, f\right)-\int_{r_{n}}^{R_{n}} \frac{n(t, 0, f)}{t} d t \\
& =N\left(R_{n}, 0, f\right)+O\left(\log R_{n}\right) .
\end{aligned}
$$

This implies together with (iii) that

$$
N\left(r_{n}, 0, f\right)=(1+o(1)) T\left(R_{n}, 0, f\right),
$$


and since $N\left(r_{n}, \infty, f\right) \leqq N\left(R_{n}, \infty, f\right)$, we see from (i) and (ii) that

$$
\log |f(z)| \geqq\left(\frac{1}{2}+o(1)\right) \Delta(\infty, f) T\left(R_{n}, f\right)
$$

on $|z|=r_{n}$. Since $f$ is non-rational, it follows from (iv) that there exists a sequence $K_{n}$ with $\lim K_{n}=\infty$ such that

$$
\log |f(z)| \geqq K_{n}^{2} \log r_{n}
$$

on $|z|=r_{n}$.

We choose $\varrho_{n}<r_{n}$ such that $|f(z)|>1$ in $\varrho_{n}<|z| \leqq r_{n}$ and that there exists a point $\zeta_{n}$ lying on $|z|=\varrho_{n}$ such that $\left|f\left(\zeta_{n}\right)\right|=1$. On the boundary of the annulus $H_{n}: \varrho_{n}<|z|<r_{n}$ we have

$$
\log |f(z)| \geqq K_{n}^{2} \log r_{n} \frac{\log \left(|z| \mid \varrho_{n}\right)}{\log \left(r_{n} / \varrho_{n}\right)},
$$

and from the superharmonicity of $\log |f(z)|$ we conclude that (vi) holds in $H_{n}$. Let $t$ be defined by the equation

$$
\frac{\log \left(t / \varrho_{n}\right)}{\log \left(r_{n} / \varrho_{n}\right)}=\frac{1}{K_{n} \log r_{n}}
$$

and let $z_{n}$ be the point on $|z|=t$ which satisfies $\arg z_{n}=\arg \zeta_{n}$. Then it follows from (vi) that $\log \left|f\left(z_{n}\right)\right| \geqq K_{n}$, and for large values of $n$ we get

Applying Lemma 2, we deduce that

$$
\left|z_{n}-\zeta_{n}\right| \leqq \frac{2\left|\zeta_{n}\right|}{K_{n}}
$$

$$
U\left(\zeta_{n}, \frac{2 K\left|\zeta_{n}\right|}{K_{n}}\right)
$$

contains at least two points of $E$ for all large $n$. This is a contradiction with (C), and Theorem 4 is proved.

\section{Proof of Theorem 5}

Let $\varphi(r)$ be an increasing function such that $\varphi(r) \rightarrow \infty$ as $r \rightarrow \infty$. We denote by $(a, b)$ the open segment $a<x<b$ on the positive real axis. We set

$$
f_{n}(z)=\prod_{p=1}^{n}\left(1-\frac{z}{1+(p / n)}\right)^{2}
$$

and let $x_{p}, p=1,2, \ldots, 2 n-1$, be the roots of the equation $f_{n}^{\prime}(z)=0$ arranged such that $x_{p+1}>x_{p}$. We see easily that

$$
x_{1}=1+\frac{1}{n}<x_{2}<x_{3}=1+\frac{2}{n}<x_{4}<\ldots<x_{2 n-2}<x_{2 n-1}=2 .
$$


We denote

$$
d_{n}=\min \left\{f_{n}\left(x_{2 p}\right): p=1,2, \ldots, n-1\right\} .
$$

Then $d_{n}>0$, and we note that if $0<b \leqq d_{n} / 4$ and the points $y_{s}, s=1,2, \ldots, 2 n$, are chosen such that $b / 2<f\left(y_{s}\right)<2 b, y_{1} \in\left(0, x_{1}\right), y_{s} \in\left(x_{s-1}, x_{s}\right)$ for $s=2,3, \ldots, 2 n-1$, and $y_{2 n} \in(2,3)$, then

$$
\min \left\{\left|y_{s^{-}} x_{p}\right|: p=1,2, \ldots, 2 n-1, s=1,2, \ldots, 2 n\right\} \geqq \alpha(n, b)
$$

for some $\alpha(n, b)>0$ depending only on $n$ and $b$.

We set $t_{k}=k$ ! and the sequences $r_{k}$ and $\varrho_{k}$ of positive real numbers are chosen such that

$$
t_{k} \leqq \log \log \log r_{k}
$$

and

$$
r_{k}<\log \varrho_{k}<\log \log \log r_{k+1}
$$

for every $k$. We set

$$
f(z)=\prod_{k=1}^{\infty}\left(f_{t_{k}}\left(z / r_{k}\right)\left(1-z / \varrho_{k}\right)^{-2 t_{k}}\right)
$$

We may write

$$
f(z)=\frac{\prod_{s=1}^{\infty}\left(1-z / z_{s}\right)}{\prod_{s=1}^{\infty}\left(1-z / b_{s}\right)},
$$

where the sequences $z_{s}$ and $b_{s}$ are increasing.

We denote $n_{k}=n\left(r_{k} / 2,0, f\right)$. Then $n\left(r_{k} / 2, \infty, f\right)=n_{k}$, and we may assume that the sequences $r_{s}$ aidd $\varrho_{s}$ are chosen such that if $\sqrt{r_{k}}<|z|<r_{k}^{2}$ and $k \geqq 2$, then

$$
f(z)=(1+o(1)) f_{t_{k}}\left(z / r_{k}\right) A_{k},
$$

where

$$
A_{k}=\prod_{s=1}^{n_{k k}} \frac{b_{s}}{z_{s}}>\frac{4}{d_{t_{k}}}
$$

and $o(1)$ satisfies $|o(1)|<1 / 100$ in $\sqrt{r_{k}}<|z|<r_{k}^{2}$.

Let $x_{p}, x_{1}<x_{2}<\ldots<x_{2 t_{k}-1}$, be the zeros of $f_{t_{k}}^{\prime}$. From (iv) and (v) we get $f\left(r_{k} / 2\right)>2, f\left(x_{2 p-1} r_{k}\right)=0$ for $p=1,2, \ldots, t_{k}, f\left(x_{2 p} r_{k}\right)>2$ for $p=1,2, \ldots, t_{k}-1$, and $f\left(3 r_{k}\right)>2$. Therefore $f$ has real 1-points $\xi_{k}, k=1, \ldots, 2 t_{k}$, such that $\xi_{1} \in\left(r_{k} / 2, x_{1} r_{k}\right), \xi_{p} \in\left(x_{p-1} r_{k}, x_{p} r_{k}\right)$ for $p=1,2, \ldots, 2 t_{k}-1$, and $\xi_{2 t_{k}} \in\left(2 r_{k}, 3 r_{k}\right)$. It follows from (iv) that the points $y_{p}=\xi_{p} / r_{k}$ satisfy

$$
\frac{1}{2 A_{k}}<f_{t_{k}}\left(y_{p}\right)<\frac{2}{A_{k}}
$$

and we conclude from (i) and (v) that

$$
\left|x_{p}-y_{s}\right| \geqq \alpha\left(t_{k}, 1 / A_{k}\right)
$$

for all $p$ and $s$. Since $|f(z)| \geqq 5$ on the circles $|z|=\sqrt{r_{k}}$ and $|z|=\sqrt{r_{k+1}}$, it follows from Rouche's theorem that $f$ has exactly $2 t_{k}$ 1-points in $\sqrt{r_{k}} \leqq|z| \leqq \sqrt{r_{k+1}}$, and 
we deduce that the only 1 -points of $f$ lying in $\sqrt{r_{k}} \leqq|z| \leqq \sqrt{r_{k+1}}$ are the points $\xi_{p}, p=1,2, \ldots, 2 t_{k}$.

Let $E=\left\{a_{n}\right\}$ be the set of the zeros, 1-points and poles of $f$. Then $E$ lies on the positive real axis. We assume that $E$ is arranged such that $0=a_{1}<a_{2}<a_{3}<\ldots$. It follows from (vi) that those points $a_{n}$ which lie in $\sqrt{r_{k}} \leqq|z| \leqq \sqrt{r_{k+1}}$ satisfy

$$
a_{n+1}>a_{n}\left(1+\frac{1}{4} \alpha\left(t_{k}, 1 / A_{k}\right)\right) \text {. }
$$

Since the value of $A_{k}$ does not depend on the choice of $r_{k}$, we may assume that $r_{k}$ is chosen so large that

$$
\alpha\left(t_{k}, 1 / A_{k}\right) \geqq\left(\varphi\left(\sqrt{r_{k}}\right)\right)^{-1 / 2}
$$

Then $E$ satisfies (D) for all large values of $n$.

If $n$ is large, then

and

$$
N\left(r_{k}^{3}, 0, f\right) \geqq t_{k} \log r_{k}
$$

$$
N\left(r_{k}^{3}, \infty, f\right) \leqq(6+o(1)) t_{k-1} \log r_{k} .
$$

Since $t_{k-1}=t_{k} / k=o\left(t_{k}\right)$, we deduce now that $\Delta(\infty, f)=1$. This implies that the set $E$ does not belong to the class $P(V)$, and Theorem 5 is proved.

\section{Proof of Theorem 6}

Let $U\left(a_{n}, d_{n}\right)$ be as in Theorem 6 . Taking a subset of the union of the discs $U\left(a_{n}, d_{n}\right)$, if necessary, we may assume that $\left|a_{1}\right|>100,\left|a_{n}^{2}\right|<\left|a_{n+1}\right|$ and $0<d_{n}<1$ for all $n$. We set

$$
f(z)=\prod_{n=1}^{\infty}\left(1-\left(\frac{d_{n}}{2\left(z-a_{n}\right)}\right)^{t_{n}}\right),
$$

where the sequence $t_{n}$ grows at least so rapidly that $|f(z)|<2$ outside the union of the discs $U\left(a_{n}, d_{n}\right)$. Furthermore, we assume that

for $n \geqq 2$. We have

$$
2 n t_{n-1} \log \left|a_{n}\right|<\frac{1}{8} t_{n} \log \frac{\left|a_{n}\right|}{\left|a_{n}\right|-d_{n} / 8}
$$

$$
\begin{aligned}
N\left(\left|a_{n}\right|, 0, f\right) & \geqq N\left(\left|a_{n}\right|, 0, f\right)-N\left(\left|a_{n}\right|-d_{n} / 8,0, f\right) \\
& \geqq n\left(\left|a_{n}\right|-d_{n} / 8,0, f\right) \log \frac{\left|a_{n}\right|}{\left|a_{n}\right|-d_{n} / 8} \\
& \geqq \frac{1}{8} t_{n} \log \frac{\left|a_{n}\right|}{\left|a_{n}\right|-d_{n} / 8},
\end{aligned}
$$


and

$$
N\left(\left|a_{n}\right|, \infty, f\right) \leqq n\left(\left|a_{n}\right|-1, \infty, f\right) \log \left|a_{n}\right| \leqq 2 t_{n-1} \log \left|a_{n}\right|,
$$

and these estimates imply together with (i) that

$$
N\left(\left|a_{n}\right|, \infty, f\right) \leqq \frac{1}{n} N\left(\left|a_{n}\right|, 0, f\right)
$$

for $n \geqq 2$. Therefore $\Delta(\infty, f)=1$, and since $f$ is bounded in the complement of the union of the discs $U\left(a_{n}, d_{n}\right)$, it omits at least three values outside the discs $U\left(a_{n}, d_{n}\right)$. Therefore the set

$$
\bigcup_{n=1}^{\infty} U\left(a_{n}, d_{n}\right)
$$

cannot belong to the class $P(V)$, and Theorem 6 is proved.

\section{Proof of Theorem 7}

Let $f$ be transcendental and meromorphic in the plane and and let the set $E=\left\{a_{n}\right\}=f^{-1}(\{0,1, \infty\})$ satisfy the condition $\lim \left|a_{n+1} / a_{n}\right|=\infty$.

We denote

$$
\begin{aligned}
& \gamma_{n}=\left\{z:|z|=\sqrt{\left|a_{n} a_{n+1}\right|},\right. \\
& s_{n}=\left\{z:|z|=\left|a_{n}\right| / 2\right\}, \\
& S_{n}=\left\{z:|z|=2\left|a_{n}\right|\right\},
\end{aligned}
$$

and let $D_{n}$ be the annulus which is bounded by $\gamma_{n-1}$ and $\gamma_{n}$.

It follows from Lemma 1, applied in the annuli $2\left|a_{n}\right|<|z|<\left|a_{n+1}\right| / 2$, that there exists a sequence $U\left(b_{n}, d_{n}\right)$ such that $\lim d_{n}=0$ and that $f\left(\gamma_{n}\right) \subset U\left(b_{n}, d_{n}\right)$ for all large $n$. It does not mean any restriction to assume that the sequence $d_{n}$ is decreasing.

Let $n_{0}$ be so large that $d_{n_{0}}<1 / 100$. Let $n>n_{0}$ and let us suppose that

$$
U\left(b_{n-1}, d_{n-1}\right) \cap U\left(b_{n}, d_{n}\right)=\emptyset .
$$

Joining $\gamma_{n-1}$ to $\gamma_{n}$ by a path $\gamma \subset D_{n}$ we see that $f$ takes in $D_{n}$ at least one value lying outside the union of the discs $U\left(b_{n}, d_{n}\right)$ and $U\left(b_{n-1}, d_{n-1}\right)$. Since the image of the boundary of $D_{n}$ is contained in this union, we deduce that $f$ takes in $D_{n}$ all values lying in the complement of this union. This is possible only in the case that for some combination $\left\{w_{1}, w_{2}, w_{3}\right\}=\{0,1, \infty\}$ we have $w_{1} \in U\left(b_{n-1}, d_{n-1}\right)$, $f\left(a_{n}\right)=w_{2}$, and $w_{3} \in U\left(b_{n}, d_{n}\right)$. We note that $U\left(b_{n-1}, d_{n-1}\right) \subset U\left(w_{1}, 2 d_{n-1}\right)$. Let us suppose that

$$
U\left(b_{n-1}, d_{n-1}\right) \cap U\left(b_{n}, d_{n}\right) \neq \emptyset .
$$

In this case the union of the $\operatorname{discs} U\left(b_{n-1}, d_{n-1}\right)$ and $U\left(b_{n}, d_{n}\right)$ contains at most one 
of the values 0,1 and $\infty$, and since $f$ omits at least two of these values in $D_{n}$, we conclude from the maximum principle that $f\left(D_{n}\right)$ is contained in the union of these discs. We note that

$$
f\left(D_{n}\right) \subset U\left(b_{n-1}, d_{n-1}\right) \cup U\left(b_{n}, d_{n}\right) \subset U\left(f\left(a_{n}\right), 4 d_{n-1}\right) .
$$

Combining the estimations above, we conclude that, if $n>n_{0}$, then $f\left(\gamma_{n}\right) \subset U\left(c_{n}, 4 d_{n}\right)$, where $c_{n} \in\{0,1, \infty\}$, and just one of the following two cases for $D_{n}$ occurs:

$$
\begin{gathered}
\left\{c_{n-1}, f\left(a_{n}\right), c_{n}\right\}=\{0,1, \infty\}, \quad \text { or } \\
f\left(D_{n}\right) \subset U\left(f\left(a_{n}\right), 4 d_{n-1}\right) \text { and } c_{n-1}=f\left(a_{n}\right)=c_{n} .
\end{gathered}
$$

Let us suppose that the case (ii) happens for all large $n$, say for $n \geqq n_{1}>n_{0}$. Then we have

$$
c_{n_{1}-1}=f\left(a_{n_{1}}\right)=c_{n_{1}}=f\left(a_{n_{1}+1}\right)=c_{n_{1}+1}=f\left(a_{n_{1}+2}\right)=\ldots,
$$

and we see that the image of the set $|z| \geqq\left|a_{n_{1}}\right|$ is contained in $U\left(f\left(a_{n_{1}}\right), 4 d_{n_{1}-1}\right)$. This is impossible, and we conclude that there exist arbitrarily large values of $n$ such that the case (i) happens.

Let the case (i) occur for $D_{n}$ with $n>n_{0}$. We assume first that $c_{n-1}=0, f\left(a_{n}\right)=1$ and $c_{n}=\infty$. Let us suppose that $a_{n}$ is a multiple root of the equation $f(z)=1$. Let $J$ be the segment on the positive real axis which joins the points 0 and 1 . Then there exists a region $G \subset D_{n}$ such that the boundary of $G$ is contained in $\gamma_{n-1} \cup$ $f^{-1}(J)$ and that $a_{n}$ is a boundary point of $G$. Then the image of the boundary of $G$ is contained in $J \cup U\left(0,4 d_{n-1}\right)$, and since $f$ takes in $G$ near the point $a_{n}$ at least one value lying outside $J \cup U\left(0,4 d_{n-1}\right)$, we conclude that $f$ takes in $G$ all values lying outside $J \cup U\left(0,4 d_{n-1}\right)$. This implies that $f$ takes the value $\infty$ in $D_{n}$, and we are led to a contradiction. Therefore $a_{n}$ is a simple 1-point of $f$. Since $f$ has no zeros or poles in $\left|z-a_{n}\right| \leqq\left|a_{n}\right| / 2$, we conclude from the maximum and minimum principles that $|f(z)|$ takes the value 1 at some point of $\left|z-a_{n}\right|=\left|a_{n}\right| / 2$. Applying Schottky's theorem, we see that there exists an absolute constant $q>0$ such that $|f(z)|>q$ on $s_{n}$ and $|f(z)|<1 / q$ on $S_{n}$. Then it follows from Rouche's theorem that $n\left(\left|a_{n}\right| / 2, b\right)=n\left(\left|a_{n}\right| / 2,0\right)$ for $b \in U(0, q)$ and $n\left(2\left|a_{n}\right|, b\right)=n\left(2\left|a_{n}\right|, \infty\right)$ for $b \in U(\infty, q)$. Modifying these results for the general case, we get the following conclusion: If the case (i) happens for $D_{n}$ and $n>n_{0}$, then $a_{n}$ is a simple root of the equation $f(z)=f\left(a_{n}\right)$ and

$$
n\left(\left|a_{n}\right| / 2, b\right)=n\left(\left|a_{n}\right| / 2, c_{n-1}\right)
$$

for $b \in U\left(c_{n-1}, q\right)$, and

$$
n\left(2\left|a_{n}\right|, b\right)=n\left(2\left|a_{n}\right|, c_{n}\right)
$$

for $b \in U\left(c_{n}, q\right)$. Here $q>0$ is an absolute constant.

We denote by $t_{n}$ the radius of the circle $\gamma_{n}$. Let the case (i) happen for $n, n>n_{0}$, and let $p>n$ be the smallest integer such that the case (i) happens for $p$, too. In order to simplify the notations, we assume that $c_{n-1}=0, f\left(a_{n}\right)=1$ and $c_{n}=\infty$. 
It follows from (ii) that $c_{p-1}=\infty$ and that the image of the set $t_{n} \leqq|z| \leqq t_{p-1}$ is contained in $U\left(\infty, 4 d_{n}\right)$. Furthermore, we deduce from (i) that $\left\{f\left(a_{p}\right), c_{p}\right\}=\{0,1\}$.

We denote $k_{n}=n\left(t_{n}, 1\right)$. Applying Rouche's theorem, we get

and we conclude that

$$
n\left(t_{n-1}, 0\right)=n\left(t_{n}, 0\right)=n\left(t_{n}, 1\right)=k_{n},
$$

$$
n(r, 0)=n(r, 1)=k_{n}
$$

for $\left|a_{n}\right| \leqq r<\left|a_{p}\right|$.

Let $\left|a_{n}\right| \leqq r<\left|a_{p}\right|$. Let us suppose first that $w \notin U(0, q) \cup U(1, q)$. We apply Rouche's theorem repeatedly, and conclude that

$$
n(r, w) \geqq n\left(t_{n-1}, w\right)=n\left(t_{n-1}, 1\right)=k_{n}-1,
$$

and

$$
n(r, w) \leqq n\left(t_{p}, w\right)=n\left(t_{p}, f\left(a_{p}\right)\right)=k_{n}+1
$$

because of (v). Combining (vi) and (vii) we conclude that

$$
\left|n(r, w)-k_{n}\right| \leqq 1
$$

for $w \notin U(0, q) \cup U(1, q)$. Let us suppose now that $w \in U(0, q)$. Then it follows from (iii) that

$$
n(r, w) \geqq n\left(\left|a_{n}\right| / 2, w\right)=n\left(\left|a_{n}\right| / 2,0\right)=n\left(t_{n-1}, 0\right)=k_{n} .
$$

If $f\left(a_{p}\right)=0$, we get

$$
n(r, w) \leqq n\left(t_{p}, w\right)=n\left(t_{p}, 0\right)=k_{n}+1,
$$

and if $f\left(a_{p}\right)=1$, it follows from (iv) that

$$
n(r, w) \leqq n\left(2\left|a_{p}\right|, w\right)=n\left(2\left|a_{p}\right|, 0\right)=k_{n} .
$$

Combining the estimates (ix), (x) and (xi), we deduce that (viii) holds for $w \in U(0, q)$. By a similar consideration, we conclude that (viii) holds for $w \in U(1, q)$, too.

Since (viii) is valid for all $w \in \Sigma$ and for all $r,\left|a_{n}\right| \leqq r<\left|a_{p}\right|$, we conclude that for any large $r$, there exists a positive integer $k(r)$ such that

$$
|n(r, w)-k(r)| \leqq 1
$$

for all $w \in \Sigma$. This implies that

$$
\limsup _{r \rightarrow \infty}\left(\sup _{w \in \Sigma} n(r, w)-\inf _{w \in \Sigma} n(r, w)\right) \leqq 2 .
$$

It follows from (v) that two of the numbers $n(r, 0), n(r, 1)$ and $n(r, \infty)$ are equal to $k(r)$ and that the third of these numbers satisfies (xii) for all large $r$. This implies that

$$
\left|n\left(r, w_{1}\right)-n\left(r, w_{2}\right)\right| \leqq 1
$$

if $r \geqq r_{0}$ and $\left\{w_{1}, w_{2}\right\} \subset\{0,1, \infty\}$. 
Let $a$ be any complex value different from 0,1 and $\infty$, and let $n$ be so large that $a$ cannot belong to any of the discs $U\left(0,4 d_{n}\right), U\left(1,4 d_{n}\right)$ and $U\left(\infty, 4 d_{n}\right)$. If the case (ii) happens, then

(xiv)

$$
n(r, a)=n\left(r, w_{1}\right)=n\left(r, w_{2}\right)
$$

for $t_{n-1} \leqq r \leqq t_{n},\left\{w_{1}, w_{2}\right\}=\{0,1, \infty\}-\left\{f\left(a_{n}\right)\right\}$. If the case (i) happens and $t_{n-1} \leqq$ $r \leqq t_{n}$, then we see from (v) that

and

$$
n(r, a) \leqq n\left(t_{n}, a\right)=n\left(t_{n}, f\left(a_{n}\right)\right)=n\left(r, c_{n-1}\right)
$$

$$
n(r, a) \geqq n\left(t_{n-1}, a\right)=n\left(t_{n-1}, f\left(a_{n}\right)\right)=n\left(r, c_{n}\right) .
$$

Since $n\left(r, c_{n-1}\right)=1+n\left(r, c_{n}\right)$, we conclude that either $n(r, a)=n\left(r, c_{n}\right)$ or $n(r, a)=$ $n\left(r, c_{n-1}\right)$. This implies together with (xiv) that for all large values of $r$, there exists $w(r, a) \in\{0,1, \infty\}$ such that $n(r, a)=n(r, w(r, a))$. If $a \in\{0,1, \infty\}$, we set $w(r, a)=a$ for all $r$.

Let $a$ and $b$ be two complex values. Then we get for all large $r$,

$$
|n(r, a)-n(r, b)|=|n(r, w(r, a))-n(r, w(r, b))|,
$$

and we deduce from (xiii) that $|n(r, a)-n(r, b)| \leqq 1$. This implies that

$$
\limsup _{r \rightarrow \infty}|n(r, a)-n(r, b)| \leqq 1,
$$

which completes the proof of Theorem 7 .

\section{Proof of Theorem 8}

Let $f$ and $E$ be as in Theorem 8. Contrary to the assertion of Theorem 8, let us suppose that

$$
\lim _{n \rightarrow \infty}\left|a_{n+1} / a_{n}\right|=\infty .
$$

Let $\gamma_{n}, D_{n}$ and $U\left(b_{n}, d_{n}\right)$ be as in the proof of Theorem 7. If $n$ is large, then the set

$$
A_{n}=U\left(b_{n-1}, d_{n-1}\right) \cup U\left(b_{n}, d_{n}\right)
$$

contains at most two of the points $0,1, w_{4}$ and $\infty$. Then $f$ omits in $D_{n}$ at least one value lying in the complement of $A_{n}$, and since the image of the boundary of $D_{n}$ is contained in $A_{n}$, we conclude from the maximum principle that $f\left(D_{n}\right) \subset A_{n}$. Since $D_{n}$ is a connected set, $f\left(D_{n}\right)$ is connected, and we deduce that

$$
U\left(b_{n-1}, d_{n-1}\right) \cap U\left(b_{n}, d_{n}\right) \neq \emptyset .
$$

This implies together with the fact that $f\left(a_{n}\right) \in A_{n}$ that

$$
f\left(D_{n}\right) \subset A_{n} \subset U\left(f\left(a_{n}\right), 4 d_{n}\right)
$$


for all large $n$, and in the same manner as in the proof of Theorem 7, we see now that if $n$ is large, then the image of the set $|z|>\left|a_{n}\right|$ is contained in $U\left(f\left(a_{n}\right), 4 d_{n}\right)$. This is impossible, and therefore we may conclude that

This proves Theorem 8 .

$$
\liminf _{n \rightarrow \infty}\left|a_{n+1} / a_{n}\right|<\infty \text {. }
$$

\section{Proof of Theorem 9}

Let $M>1, r_{1}=e$ and $r_{n-1}=\log \log r_{n}$ for $n \geqq 2$. We set

$$
f(z)=z \prod_{n=1}^{\infty}\left(1-z / r_{n}\right)^{2(-1)^{n}} .
$$

It follows from the considerations made in [11, p. 16] that all except a finite number of the 1-points and $M$-points of $f$ lie on the positive real axis on the union of the segments $I_{n}: r_{n}^{3 / 2} \leqq x \leqq r_{n}^{3}$, and that if $n$ is large, then $I_{n}$ contains exactly one 1-point and one $M$-point of $f$. Let these points be $b_{n}$ and $z_{n}$, arranged such that $b_{n}<z_{n}$. If $x \in I_{n}$, then the logarithmic derivative of $f$ satisfies

and we get

$$
\frac{f^{\prime}(x)}{f(x)}=(1+o(1)) \frac{(-1)^{n}}{x}
$$

$$
\begin{aligned}
\log M & =\left|\log f\left(z_{n}\right)-\log f\left(b_{n}\right)\right|=\left|\int_{b_{n}}^{z_{n}} \frac{f^{\prime}(x)}{f(x)} d x\right| \\
& =(1+o(1)) \int_{b_{n}}^{z_{n}} \frac{d x}{x}=(1+o(1)) \log \left(z_{n} / b_{n}\right) .
\end{aligned}
$$

This implies that

$$
z_{n} / b_{n}=M^{1+o(1)} \rightarrow M
$$

as $n \rightarrow \infty$. Since $r_{n}^{3 / 2}<b_{n}<z_{n}<\sqrt{r_{n+1}}$ for all large $n$, we conclude from (i) that the set $E$ defined by (F) satisfies the condition (G). This proves Theorem 9.

\section{Two lemmas}

Lemma 5. Let $f$ be meromorphic in the half disc

$$
D=\{z:|z| \leqq r, \operatorname{Im} z \geqq 0\}
$$

and satisfy $|f(z)| \geqq 1$ there. There exists an absolute constant $K_{1}>0$ such that

$$
\log |f(z)| \geqq \frac{K_{1}}{r} \int_{-r / 2}^{r / 2} \log |f(x)| d x
$$

for any $z \in U(i r / 2, r / 8)$. 
Proof. Let $z(w)$ map the unit disc $|w|<1$ conformally on to $D$ such that $w(0)=$ $i r / 2$. From the superharmonicity of $\log |f(z(w))|$ it follows that

$$
\log \left|f\left(z\left(\varrho e^{i \alpha}\right)\right)\right| \geqq \frac{1}{2 \pi} \int_{0}^{2 \pi} \log \left|f\left(z\left(e^{i \varphi}\right)\right)\right| \frac{1-\varrho^{2}}{1-2 \varrho \cos (\varphi-\alpha)+\varrho^{2}} d \varphi
$$

for $\varrho<1$. Since there exist absolute constants $m_{1}>0$ and $m_{2}<1$ such that

$$
\left|\frac{d z(w)}{d w}\right| \leqq m_{1} r
$$

if $z$ lies on the segment $[-r / 2, r / 2]$, and $|w| \leqq m_{2}$ if $z(w) \in U(i r / 2, r / 8)$, it follows from (i) that

$$
\log |f(\zeta)| \geqq \frac{1-m_{2}}{2 \pi\left(1+m_{2}\right) m_{1} r} \int_{-r / 2}^{r / 2} \log |f(x)| d x
$$

for $\zeta \in U(i r / 2, r / 8)$, and Lemma 5 is proved.

Lemma 6. Let $u$ be harmonic in the annulus $H: r<|z|<R$, non-negative and continuous on its closure, and $u(z)=0$ on $|z|=r$. Let

Then

$$
\mu(R, u)=\frac{1}{2 \pi} \int_{0}^{2 \pi} u\left(R e^{i \varphi}\right) d \varphi .
$$

and

$$
u(z) \leqq \mu(R, u) \frac{R+|z|}{R-|z|}
$$

$$
u(z) \geqq \mu(R, u)\left(\frac{R-|z|}{R+|z|}-\frac{(R+r) \log (R /|z|)}{(R-r) \log (R / r)}\right)
$$

for all $z \in H$, and if $R \geqq r e^{36}$, then

$$
u(z) \geqq \frac{1}{6} \mu(R, u)
$$

for those $z$ which lie in $R / 4 \leqq|z| \leqq R / 2$.

Proof. The function

$$
v\left(\varrho e^{i \alpha}\right)=\frac{1}{2 \pi} \int_{0}^{2 \pi} u\left(R e^{i \varphi}\right) \frac{R^{2}-\varrho^{2}}{R^{2}-2 R \varrho \cos (\varphi-\alpha)+\varrho^{2}} d \varphi
$$

is harmonic in $|z|<R$, continuous on its closure, and $v(z)=u(z)$ on $|z|=R$. On $|z|=r$ we have $u(z)=0 \leqq v(z)$, and since

$$
v(z) \leqq \mu(R, u) \frac{R+|z|}{R-|z|}
$$


in $|z|<R$, (5) follows from the maximum principle. We see from (i) that

$$
u(z) \geqq v(z)-\mu(R, u) \frac{(R+r) \log (R /|z|)}{(R-r) \log (R / r)}
$$

on the boundary of $H$, and we conclude from the maximum principle that (ii) holds in $H_{n}$. Since

$$
v(z) \geqq \mu(R, u) \frac{R-|z|}{R+|z|}
$$

in $|z|<R$, we get (6) from (ii). The condition (7) is a direct consequence of (6). Lemma 6 is proved.

\section{Proof of Theorem 10}

Contrary to the assertion of Theorem 10, let us suppose that there exist $\alpha, \beta, E, d_{n}$ and $S$ as in Theorem 10 and a transcendental meromorphic function $f$ with $\delta(\infty, f)>0$ such that

$$
f^{-1}\left(\left\{0,1, w_{3}\right\}\right) \subset U\left(0, r_{0}\right) \cup S
$$

for some $r_{0}, w_{3}$ being different from 0 and 1 .

It follows from (H) that the number of the $a_{n}$ which lie in the annulus $r<|z|<2 r$ is at most $4(\log r)^{2 \alpha}$. Therefore, if $r$ is large, we may choose $\varrho, r<\varrho<2 r$, such that

$$
\left\{z: \varrho-\frac{\varrho}{16(\log \varrho)^{2 \alpha}}<|z|<\varrho+\frac{\varrho}{16(\log \varrho)^{2 \alpha}}\right\} \cap S=\emptyset .
$$

Since $f$ is transcendental and $\delta(\infty, f)>0$, we have

$$
\frac{1}{2 \pi} \int_{0}^{2 \pi} \log ^{+}\left|f\left(r e^{i \varphi}\right)\right| d \varphi>10 \log r
$$

for all large $r$. This implies that we may choose $\varphi_{1}$ and $\varphi_{2}, \quad \varphi_{2}=\varphi_{1}+(\log \varrho)^{-3}$, such that

$$
\int_{\varphi_{1}}^{\varphi_{2}} \log ^{+}\left|f\left(\varrho e^{i \varphi}\right)\right| d \varphi>10(\log \varrho)^{-2} .
$$

We set $g(\zeta)=f\left(e^{\zeta}\right)$. It follows from (ii) that

$$
\int_{\varphi_{1}}^{\varphi_{2}} \log ^{+}|g(\log \varrho+i \varphi)| d \varphi \geqq 10(\log \varrho)^{-2} .
$$

We denote $E_{\zeta}=\left\{\zeta: e^{\zeta} \in E\right\}$. We see from $(\mathrm{H})$ that if $b \in E_{\zeta}$ and $\operatorname{Re} b$ is large, then

$$
\left\{\zeta: 0<|\zeta-b|<\frac{1}{2(\operatorname{Re} b)^{\alpha}}\right\} \cap E_{\zeta}=\emptyset,
$$


and from (I) we deduce that $g$ omits the values 0,1 and $w_{3}$ in

$$
G=\left\{\zeta: \operatorname{Re} \zeta \geqq \gamma_{0}\right\}-\bigcup_{b \in E_{\zeta}} U(b, d(b))
$$

if $\gamma_{0}>0$ is chosen sufficiently large and $d(b)$ is defined by the equation

$$
\log \frac{1}{d(b)}=\frac{1}{2}(\operatorname{Re} b)^{2+\beta} .
$$

We assume that $\log \varrho>100+\gamma_{0}$, and we conclude from (i) that

$$
\left\{\zeta:|\operatorname{Re} \zeta-\log \varrho| \subset \frac{1}{32(\log \varrho)^{2 \alpha}}\right\} \subset G .
$$

Let $J$ be the segment $\operatorname{Re} \zeta=\log \varrho, \varphi_{1} \leqq \operatorname{Im} \zeta \leqq \varphi_{2}$. It follows from (iii) that there exists $\zeta_{0} \in J$ such that $\left|g\left(\zeta_{0}\right)\right| \geqq 1$. Since

$$
U\left(\zeta_{0}, \frac{1}{32(\log \varrho)^{2 \alpha}}\right) \subset G
$$

we deduce from Schottky's theorem that there exists $M_{5}>0$ depending only on $w_{3}$ such that $|g(\zeta)| \geqq M_{5}$ in

Applying Lemma 5 in the half disc

$$
U\left(\zeta_{0}, \frac{1}{64(\log \varrho)^{2 \alpha}}\right) .
$$

$$
D=\left\{\zeta: \operatorname{Re} \zeta \leqq \log \varrho,\left|\zeta-(i / 2)\left(\varphi_{1}+\varphi_{2}\right)-\log \varrho\right| \leqq(\log \varrho)^{-3}\right\},
$$

we conclude now from (iii) that there exists $\zeta_{1} \in D$ such that in $\left|\zeta-\zeta_{1}\right| \leqq(\log \varrho)^{-5}$.

$$
\log |g(\zeta)| \geqq K_{1} \log \varrho
$$

Let $b \in E_{\zeta} \cap\{\zeta:(3 / 4) \log \varrho<\operatorname{Re} \zeta<(4 / 3) \log \varrho\}$. It follows from (iv) that

$$
U\left(b, \frac{1}{3(\operatorname{Re} b)^{\alpha}}\right)-U(b, d(b)) \subset G .
$$

We choose $d, 0<d<1 / 1000$, such that the set $U(a, 8 d)$ cannot contain two of the points 0,1 and $w_{3}$ for any $a \in \Sigma$. We see from Lemma 1 and (vii) that there exists $M_{6}>0$ depending only on $w_{3}$ such that the image of the circle

$$
\Gamma_{b}=\left\{\zeta:|\zeta-b|=\frac{1}{M_{6}(\operatorname{Re} b)^{\alpha}}\right\}
$$

is contained in some set $U\left(w_{1}(b), d\right)$ and the image of

$$
\gamma_{b}=\left\{\zeta:|\zeta-b|=M_{6} d(b)\right\}
$$

is contained in some $U\left(w_{2}(b), d\right)$. If

$$
U\left(w_{1}(b), d\right) \cap U\left(w_{2}(b), d\right)=\emptyset,
$$


then $g$ would take at least one of the values 0,1 and $w_{3}$ in

$$
D_{b}=\left\{\zeta: M_{6} d(b) \leqq|\zeta-b| \leqq \frac{1}{M_{6}(\operatorname{Re} b)^{\alpha}}\right\} .
$$

This is impossible, and we deduce that there exists $w(b) \in \Sigma$ such that

$$
f\left(D_{b}\right) \subset U(w(b), 2 d) .
$$

Let $C_{b}$ be the $\operatorname{disc}|\zeta-b|<(\log \varrho)^{-3}$. Let us suppose that there exists $R$, $(\log \varrho)^{-5}<R<100$, such that $|g(\zeta)| \geqq 2$ in

$$
\left\{\zeta:(\log \varrho)^{-6} \leqq\left|\zeta-\zeta_{1}\right| \leqq R\right\}-\bigcup_{b \in E_{\zeta}} C_{b}
$$

and that there exists $\zeta_{2}$ such that $\left|g\left(\zeta_{2}\right)\right|=2$ and that

Let

$$
\zeta_{2} \in\left\{\zeta:\left|\zeta-\zeta_{1}\right|=R\right\}-\bigcup_{b \in E_{\zeta}} C_{b}
$$

$$
E_{1}=\left\{b \in E_{\zeta}: C_{b} \cap U\left(\zeta_{1}, R\right) \neq \emptyset\right\} .
$$

It follows from (viii) that $|g(\zeta)| \geqq 1$ on $D_{b}$ for $b \in E_{1}$, and from (iv) we conclude that the number of the points of $E_{1}$ is at most

The function

$$
q=320000(\log \varrho)^{2 \alpha} \text {. }
$$

$$
\omega(\zeta)=\frac{\log \left(R /\left|\zeta-\zeta_{1}\right|\right)}{\log \left(R(\log \varrho)^{6}\right)}-\sum_{b \in E_{1}} \frac{\log (3 R /|\zeta-b|)}{\log \left(3 R /\left(M_{6} d(b)\right)\right)}
$$

is harmonic in

$$
A=\left\{\zeta:(\log \varrho)^{-6} \leqq\left|\zeta-\zeta_{1}\right| \leqq R\right\}-\bigcup_{b \in E_{1}} U\left(b, M_{6} d(b)\right),
$$

$\omega(\zeta) \leqq 1$ on $\left|\zeta-\zeta_{1}\right|=(\log \varrho)^{-6}$, and $\omega(\zeta) \leqq 0$ at the other boundary points of $A$. Since $|g(\zeta)| \geqq 1$ in $A$, it follows from (vi) that

$$
\log |g(\zeta)| \geqq K_{1} \omega(\zeta) \log \varrho
$$

on the boundary of $A$, and from the superharmonicity of $\log |g(\zeta)|$ in $A$ we conclude that (x) holds in $A$.

It follows from $(\mathrm{v})$ that $U\left(\zeta_{1},(\log \varrho)^{-2}\right) \cap E_{\zeta}=\emptyset$. Therefore we may choose $s, 1 \leqq s \leqq 2$, such that the point

$$
\zeta_{3}=\zeta_{1}+\left(\zeta_{2}-\zeta_{1}\right)\left(1-s(\log \varrho)^{-(1+\alpha) / 2}\right)
$$

lies outside the union of the discs $C_{b}$, and we deduce from the definition of $d(b)$ and (ix) that

$$
\begin{aligned}
\omega\left(\zeta_{3}\right) & \geqq \frac{1}{7(\log \varrho)^{(1+\alpha) / 2} \log \log \varrho}-O\left(\frac{q \log \log \varrho}{(\log \varrho)^{2+\beta}}\right) \\
& \geqq \frac{(\log \varrho)^{(1-\alpha) / 4}}{\log \varrho}
\end{aligned}
$$


if $\varrho$ is large. This implies together with (x) that $\left|g\left(\zeta_{3}\right)\right|>10$, and since $g\left(\zeta_{2}\right) \leqq 2$, we deduce from Lemma 2 that there exists $b \in E_{\zeta}$ such that

$$
\left|b-\zeta_{2}\right| \leqq 2 K\left|\zeta_{2}-\zeta_{3}\right| \leqq 4 K R(\log \varrho)^{-(1+\alpha) / 2} .
$$

This implies that both of the points $\zeta_{2}$ and $\zeta_{3}$ lie in

$$
U\left(b, \frac{1}{M_{6}(\operatorname{Re} b)^{\alpha}}\right)
$$

and since $\zeta_{2}$ and $\zeta_{3}$ lie outside the union of the discs $C_{b}$, we conclude that $\zeta_{3}, \zeta_{2} \in D_{b}$. This contradicts (viii) because $\left|g\left(\zeta_{2}\right)\right| \leqq 2$ and $\left|g\left(\zeta_{3}\right)\right|>10$. Therefore we deduce now that $|g(\zeta)| \geqq 2$ in

$$
U\left(\zeta_{1}, 100\right)-\bigcup_{b \in E_{\zeta}} C_{b} .
$$

Combining this with (viii) and letting $\varrho$ grow, we see that there exists $\log \varrho_{0}>0$ such that $|g(\zeta)| \geqq 1$ in

$$
\left\{\zeta: \operatorname{Re} \zeta>\log \varrho_{0}\right\}-\bigcup_{b \in E_{\zeta}} U\left(b, M_{6} d(b)\right),
$$

which, written for $f$, means that $|f(z)| \geqq 1$ in

$$
\left\{z:|z|>\varrho_{0}\right\}-\bigcup_{n=1}^{\infty} U\left(a_{n}, t_{n}\right),
$$

where the radii $t_{n}$ are chosen by the equation

$$
\log \frac{1}{t_{n}}=\frac{1}{4}\left(\log \left|a_{n}\right|\right)^{2+\beta}
$$

We choose a sequence $r_{n}, r_{1}>\left(4+\varrho_{0}\right)^{100}$, such that $r_{n-1}^{2}<r_{n}<2 r_{n-1}^{2}$, there are no poles of $f$ on $|z|=r_{n}$, and

$$
\left\{z:\left|r_{n}-\right| z||<\frac{r_{n}}{16\left(\log r_{n}\right)^{2 x}}\right\} \cap S_{0}=\emptyset,
$$

where $S_{0}$ is the union of the discs $U\left(a_{k}, t_{k}\right)$, and $x_{n}$ is chosen such that $r_{n}^{1 / 100} / 2<$ $x_{n}<r_{n}^{1 / 100}$ and that (xii) is satisfied if $r_{n}$ is replaced by $x_{n}$.

Let $u$ be the function harmonic in $B_{n}: x_{n}<|z|<r_{n}$ which satisfies $u(z)=$ $\log |f(z)|$ on $|z|=r_{n}$ and $u(z)=0$ on $|z|=x_{n}$. For $a_{k}$ lying in $B_{n}$ we set

It follows from Lemma 6 that

$$
\omega_{k}(z)=\frac{\log \left(2 r_{n} /\left|z-a_{k}\right|\right)}{\log \left(2 r_{n} / t_{k}\right)} .
$$

$$
u(z) \leqq 2 m\left(r_{n}, \infty\right) \frac{r_{n}+\left|a_{k}\right|}{r_{n}-\left|a_{k}\right|}
$$


on $\left|z-a_{k}\right|=t_{k}$, and therefore we may conclude that

$$
\log |f(z)| \geqq u(z)-2 m\left(r_{n}, \infty\right) \sum_{a_{k} \in B_{n}} \frac{r_{n}+\left|a_{k}\right|}{r_{n}-\left|a_{k}\right|} \omega_{k}(z)
$$

on the boundary of $B_{n}-S_{0}$, and from the superharmonicity of $\log |f(z)|$ it follows that (xiii) holds in $B_{n}-S_{0}$, especially on $|z|=r_{n-1}$.

Let $|z|=r_{n-1}$. If $r_{n} / 2 \leqq\left|a_{k}\right|<r_{n}$, then we see from (xii) and (xi) that

$$
\frac{r_{n}+\left|a_{k}\right|}{r_{n}-\left|a_{k}\right|} \omega_{k}(z)=O\left(\frac{\left(\log r_{n}\right)^{2 \alpha}}{\left(\log r_{n}\right)^{2+\beta}}\right)=o\left(\left(\log r_{n}\right)^{-2}\right),
$$

and since the number of these points $a_{k}$ is $O\left(\left(\log r_{n}\right)^{2 x}\right)$, we deduce that

$$
\sum_{1} \frac{r_{n}+\left|a_{k}\right|}{r_{n}-\left|a_{k}\right|} \omega_{k}(z)=o(1)
$$

where the sum $\sum_{1}$ is taken over those $a_{k}$ which lie in $r_{n} / 2 \leqq|z|<r_{n}$. If $x_{n}<\left|a_{k}\right|<r_{n} / 2$, then (xi) implies that

$$
\frac{r_{n}+\left|a_{k}\right|}{r_{n}-\left|a_{k}\right|} \omega_{k}(z)=O\left(\frac{\log r_{n}}{\left(\log r_{n}\right)^{2+\beta}}\right),
$$

and since the number of these points $a_{k}$ is $O\left(\left(\log r_{n}\right)^{1+2 \alpha}\right)$ and $\beta>2 \alpha$, we conclude that

$$
\sum_{2} \frac{r_{n}+\left|a_{k}\right|}{r_{n}-\left|a_{k}\right|} \omega_{k}(z)=o(1),
$$

where the sum $\sum_{2}$ is taken over those $a_{k}$ which lie in $x_{n}<|z|<r_{n} / 2$. Combining these estimates with (xiii), we get

$$
\log |f(z)| \geqq u(z)+o\left(m\left(r_{n}, \infty\right)\right),
$$

and using the condition (6) of Lemma 6, we get

$$
\log |f(z)| \geqq\left(\frac{98}{198}+o(1)\right) m\left(r_{n}, \infty\right)
$$

on $|z|=r_{n-1}$.

From (xiv) we conclude that

$$
m\left(r_{n}, \infty\right) \leqq \frac{199}{98} m\left(r_{n-1}, \infty\right)
$$

for all large values of $n$, say for $n \geqq p$. Then, if we write $\delta=\delta(\infty, f)$,

$$
\begin{aligned}
T\left(r_{p}^{2 k}, f\right) & \leqq T\left(r_{p+k}, f\right) \leqq \frac{2}{\delta} m\left(r_{p+k}, \infty\right) \\
& \leqq \frac{2}{\delta}\left(\frac{199}{98}\right)^{k} m\left(r_{p}, \infty\right),
\end{aligned}
$$


and we deduce that if $R_{k}=r_{p}^{2^{k}}$, then $T\left(R_{k}, f\right)=O\left(\left(\log R_{k}\right)^{9 / 8}\right)$ as $k \rightarrow \infty$. This implies that

$$
T(r, f)=O\left((\log r)^{9 / 8}\right) .
$$

We denote by $z_{k}$ and $b_{k}$ the zeros and poles of $f$, and by $u_{n}$ (resp. $v_{n}$ ) the number of zeros (resp. poles) of $f$ lying in $\left|z-a_{n}\right|<1 /\left|a_{n}\right|$. We choose $\zeta$ lying on $\left|z-a_{n}\right|=t_{n}$ such that $\left|\zeta-b_{h}\right| \geqq t_{n} / v_{n}$ for any $k$. Applying Poisson-Jensen formula with $R=2\left|a_{n}\right|$ we obtain, since $|f(\zeta)| \geqq 1$, that

(xvii)

$$
\begin{aligned}
0 & \leqq \log |f(\zeta)| \\
& \leqq 4 m(R, \infty)+\sum_{\left|z_{k}\right|<R} \log \left|\frac{R\left(\zeta-z_{k}\right)}{R^{2}-\bar{z}_{k} \zeta}\right|-\sum_{\left|b_{k}\right|<R} \log \left|\frac{R\left(\zeta-b_{k}\right)}{R^{2}-\bar{b}_{k} \zeta}\right| \\
& \leqq\left(v_{n}-u_{n}\right) \log \frac{1}{t_{n}}+O(T(R, f))+v_{n} \log v_{n}+O(n(R, \infty) \log R) .
\end{aligned}
$$

It follows from (xvi) that $n(R, \infty)=O\left((\log R)^{1 / 8}\right)$, and therefore we may conclude from (xvii) and (xi) that

$$
\left(u_{n}-v_{n}\right)(\log R)^{2} \leqq O\left((\log R)^{9 / 8}\right) .
$$

This implies that $u_{n} \leqq v_{n}$ for all large $n$, and therefore

$$
n(r, 0) \leqq O(1)+n(r, \infty)
$$

for those large values of $r$ which lie outside the union of the intervals $\left|a_{k}\right|-1 /\left|a_{k}\right|<$ $r<a_{k}+1 /\left|a_{k}\right|$. Therefore we may deduce from (xviii) that $\delta(0, f) \geqq \delta(\infty, f)$. This is impossible, since the growth condition (xvi) quarantees that $f$ has at most one deficient value. We are led to a contradiction, and Theorem 10 is proved.

\section{A lemma needed in the proof of Theorem 11}

Schottky's theorem is proved by Ahlfors in the following form.

Schottky's theorem. If $g$ is regular in $|z|<1$ and omits there the values 0 and 1 , then

$$
\log ^{+}|g(z)| \leqq \frac{1+|z|}{1-|z|}\left(7+\log ^{+}|g(0)|\right)
$$

We shall need the following

Lemma 7. Let $E$ and $d_{n}$ be as in Theorem 11 and let $f$ be transcendental and meromorphic in the plane such that $\delta(\infty, f)>0$ and that

$$
f^{-1}\left(\left\{0,1, w_{3}\right\}\right) \subset U\left(0, r_{0}\right) \cup \bigcup_{n=1}^{\infty} U\left(a_{n}, d_{n}\right)
$$


for some $r_{0}>0, w_{3}$ being different from 0 and 1 . Then there exist sequences $R_{n}$, $R_{n} \rightarrow \infty$ as $n \rightarrow \infty$, and $K_{n}, K_{n} \rightarrow \infty$ as $n \rightarrow \infty$, such that

$$
\log |f(z)| \geqq K_{n}^{2} \log R_{n}
$$

for any $z$ lying on $|z|=R_{n}$.

Proof. If $f$ satisfies $T(r, f)=O\left((\log r)^{m}\right)$ for some finite $m$, it follows from the proof of Lemma 4 that there exist large values of $r$ such that

$$
\log |f(z)| \geqq\left(\frac{1}{3}+o(1)\right) \delta(\infty, f) T(r, f)
$$

on $|z|=r$, and we may choose the desired sequences $R_{n}$ an $K_{n}$.

Let us suppose that $T(r, f) \neq O\left((\log r)^{100}\right)$. Let $r$ be large and chosen such that $m(r, \infty) \geqq(\log r)^{100}$. We choose $t, r \leqq t \leqq 2 r$, such that $U\left(t, 2 t(\log t)^{-3}\right) \cap S=\emptyset$, where

$$
S=\bigcup_{n=1}^{\infty} U\left(a_{n}, d_{n}\right)
$$

Then, if $r$ is large, $m(t, \infty)>(\log t)^{99}$.

We set $g(\zeta)=f\left(e^{\zeta}\right)$ and $S_{\zeta}=\left\{\zeta: e^{\zeta} \in S\right\}$. Since

$$
\frac{1}{2 \pi} \int_{0}^{2 \pi} \log ^{+}|g(\log t+i \varphi)| d \varphi>(\log t)^{99},
$$

we may choose $\varphi_{1}$ and $\varphi_{2}, 0 \leqq \varphi_{1} \leqq \varphi_{2}=\varphi_{1}+(\log t)^{-5} \leqq 2 \pi$, such that

$$
\int_{\varphi_{1}}^{\varphi_{2}} \log ^{+}|g(\log t+i \varphi)| d \varphi \geqq(\log t)^{92} .
$$

We may assume that $0 \leqq \varphi_{1} \leqq \pi$, for the case when $\pi \leqq \varphi_{2} \leqq 2 \pi$, is symmetric.

We choose $\varphi_{0}, \varphi_{1}<\varphi_{0}<\varphi_{2}$, such that $\left|g\left(\log t+i \varphi_{0}\right)\right|>1$. Applying Schottky's theorem in the disc

$$
U\left(\log t+i \varphi_{0},(\log t)^{-3}\right),
$$

we conclude that there exists $H_{1}>0$ depending only on $w_{3}$ such that $\log |g(\zeta)| \geqq$ $-H_{1}$ in

$$
U\left(\log t+i \varphi_{0},(\log t)^{-4}\right) .
$$

Therefore we may deduce from Lemma 5 and (i) that there exists

such that $\operatorname{Im} \zeta_{0} \geqq 0$ and

$$
\zeta_{0} \in U\left(\log t+i \varphi_{0},(\log t)^{-5}\right)
$$

$$
\log |g(\zeta)| \geqq(\log t)^{92}
$$

in the $\operatorname{disc} U\left(\zeta_{0},(2 \log t)^{-5}\right)$. 
We denote $\varrho=\operatorname{Re} \zeta_{0}$. We choose $h$ to be one of the functions $g$ and $1-g$ such that $|h(\varrho+i \pi)| \geqq 1 / 2$. We apply Schottky's theorem in the disc

to the function $1 / h$, and get

$$
U\left(\varrho+i \pi, \pi-(\log t)^{-7}\right)
$$

$$
\log |h(\zeta)| \geqq-(\log t)^{-8}
$$

for all $\zeta$ lying in

$$
U\left(\varrho+i \pi, \pi-(\log t)^{-6}\right) .
$$

Let us suppose that $\varphi_{1}<4 \pi / 5$. We denote

$$
R=\pi-\operatorname{Im} \zeta_{0}-(\log t)^{-6} .
$$

The length of the arc of the circle $|\zeta-(\varrho+i \pi)|=R$ which lies in $U\left(\zeta_{0},(2 \log t)^{-5}\right)$ is at least $(\log t)^{-6}$, and since $\log |h(\zeta)|$ is superharmonic in $U(\varrho+i \pi, R)$, we conclude from (ii) and (iii) for $\zeta=\varrho+i \pi+r e^{i \alpha}$ lying in $U(\varrho+i \pi, \pi / 10)$ that

$$
\begin{aligned}
\log |h(\zeta)| & \geqq \frac{1}{2 \pi} \int_{0}^{2 \pi} \log \left|h\left(\varrho+i \pi+R e^{i \varphi}\right)\right| \frac{\left(R^{2}-r^{2}\right) d \varphi}{R^{2}-2 r R \cos (\varphi-\alpha)+r^{2}} \\
& \geqq(\log t)^{85} .
\end{aligned}
$$

If $4 \pi / 5 \leqq \varphi_{1} \leqq \pi$, the same argument as above shows that (iv) holds on $|\zeta-(\varrho+i \pi)|=2 \pi / 5$, and then it follows from the superharmonicity of $\log |h(\zeta)|$ that (iv) is valid in $U(\varrho+i \pi, \pi / 10)$, in this case, too.

We set

Then $\omega(\zeta)$ is harmonic in

$$
\omega(\zeta)=\frac{\log \frac{\pi-(\log t)^{-6}}{|\zeta-(\varrho+i \pi)|}}{\log \log t} .
$$

$$
G=\left\{\zeta: \pi-(\log t)^{-6}>|\zeta-(\varrho+i \pi)|>\pi / 10\right\},
$$

$\omega(\zeta)=0 \quad$ on $\quad|\zeta-(\varrho+i \pi)|=\pi-(\log t)^{-6} \quad$ and $\quad \omega(\zeta)<1 \quad$ on $\quad|\zeta-(\varrho+i \pi)|=\pi / 10$. From (iv) and (iii) we deduce that

$$
\log |h(\zeta)| \geqq(\log t)^{85} \omega(\zeta)-(\log t)^{8}
$$

on the boundary of $G$, and from the superharmonicity of $\log |h(\zeta)|$ we conclude that (v) holds in $G$. Especially, if $\zeta$ lies in the disc

then

$$
A=\left\{\zeta:|\zeta-(\varrho+i \pi)|<\pi-2(\log t)^{-6}\right\},
$$

$$
\log |h(\zeta)| \geqq(\log t)^{78} .
$$

It follows from Schottky's theorem, applied in

$$
U\left(\varrho+i(\log t)^{-5}, 4(\log t)^{-4}\right),
$$


that $\log |h(\zeta)|>-\log t$ in

We set

$$
U\left(\varrho+i(\log t)^{-5}, 2(\log t)^{-4}\right) .
$$

It follows from (vi) that

$$
w(\zeta)=\frac{\log \frac{(\log t)^{-4}}{\left|\zeta-\left(\varrho+i(\log t)^{-5}\right)\right|}}{\log \log t} .
$$

$$
\log |h(\zeta)| \geqq(\log t)^{78} w(\zeta)-\log t
$$

on the boundary of the annulus

$$
(\log t)^{-5} \leqq\left|\zeta-\left(\varrho+i(\log t)^{-5}\right)\right|<(\log t)^{-4},
$$

and therefore we conclude that

$$
\log |h(\zeta)| \geqq(\log t)^{77}
$$

in $U\left(\varrho+i(\log t)^{-5},(\log t)^{-4} / 2\right)$.

Combining the estimates (vi) and (vii) we deduce that $\log |h(\zeta)| \geqq(\log t)^{77}$ on the segment

$$
\left\{\zeta: \operatorname{Re} \zeta=\varrho,-(\log t)^{-4} / 4 \leqq \operatorname{Im} \zeta \leqq 2 \pi-(\log t)^{-5}\right\} .
$$

This implies that $\log |f(z)| \geqq(\log t)^{77}$ for all $z$ lying on the circle $|z|=e^{\varrho}$, and we conclude that, if $T(r, f) \neq O\left((\log r)^{100}\right)$, then the desired sequences $R_{n}$ and $K_{n}$ exist in this case, too. This completes the proof of Lemma 7.

\section{Proof of Theorem 11}

Let $a_{n}$ and $d_{n}$ be as in Theorem 11. We denote

$$
S=\bigcup_{n=1}^{\infty} U\left(a_{n}, d_{n}\right)
$$

Contrary to the assertion of Theorem 11, let us suppose that there exists a transcendental meromorphic function $f$ such that $\delta(\infty, f)>0$ and

$$
f^{-1}\left(\left\{0,1, w_{3}\right\}\right) \subset U\left(0, r_{0}\right) \cup S
$$

for some $r_{0}>0, w_{3}$ being different from 0 and 1 .

Using Lemma 7 , we choose sequences $R_{n}, R_{n} \rightarrow \infty$ as $n \rightarrow \infty$, and $K_{n}, K_{n} \rightarrow \infty$ as $n \rightarrow \infty$, such that

$$
\log |f(z)| \geqq K_{n}^{2} \log R_{n}
$$

for any $z$ lying on $|z|=R_{n}$. 
Let $d \leqq 1 / 1000$ and $M_{6}$ be as in the proof of Theorem 10 ,

and $w_{n}$ is chosen such that

$$
\begin{aligned}
\Gamma_{n} & =\left\{z:\left|z-a_{n}\right|=\frac{\varepsilon a_{n}}{M_{6}\left(\log a_{n}\right)^{\alpha}}\right\}, \\
\gamma_{n} & =\left\{z:\left|z-a_{n}\right|=M_{6} d_{n}\right\},
\end{aligned}
$$

$$
f\left(D_{n}\right) \subset U\left(w_{n}, 2 d\right),
$$

where $D_{n}$ is the annulus bounded by $\gamma_{n}$ and $\Gamma_{n}$.

We set

$$
\omega_{k}(z)=\frac{1000+\log \left(a_{k} /\left|z-a_{k}\right|\right)}{1000+\log \left(a_{k} /\left(M_{6} d_{k}\right)\right)} .
$$

Then $\omega_{k}(z)=0 \quad$ on $\quad\left|z-a_{k}\right|=a_{k} e^{1000} \quad$ and $\quad \omega_{k}(z)=1 \quad$ on $\quad \gamma_{n}$. If $\quad\left|z-a_{k}\right| \geqq$ $a_{k}\left(\log a_{k}\right)^{-9}$, then

$$
\omega_{k}(z) \leqq \frac{19 \log \log a_{k}}{\left(\log a_{k}\right)^{2+\alpha}} .
$$

Let $z$ lie outside the union of the $\operatorname{discs} U\left(a_{s}, a_{s}\left(\log a_{s}\right)^{-9}\right)$. We denote

$$
E_{z}=\left\{a_{k}:|z| e^{-500} \leqq a_{k} \leqq|z| e^{500}\right\} .
$$

From (J) it follows that there exists $B_{1}$ depending only on $\varepsilon$ such that the number of the points of $E_{z}$ is at most $B_{1}(\log |z|)^{\alpha}$, and we conclude that there exists $B_{2}$ depending only on $\varepsilon$ such that

$$
\lambda(z)=\sum_{a_{k} \in E_{z}} \omega_{k}(z) \leqq \frac{B_{2} \log \log |z|}{(\log |z|)^{2}}
$$

if $|z|$ is sufficiently large and $z$ lies outside the union of the discs $U\left(a_{s}, a_{s}\left(\log a_{s}\right)^{-9}\right)$.

Let $k$ be fixed. Let us suppose that there exists $R, R_{k} \leqq R<R_{k} e^{37}$, such that $|f(z)| \geqq 2$ in

and that there exists

$$
\left\{z: R_{k} \leqq|z| \leqq R\right\}-\bigcup_{n=1}^{\infty} U\left(a_{n}, \frac{\varepsilon a_{n}}{M_{6}\left(\log a_{n}\right)^{\alpha}}\right)
$$

$$
z_{1} \in\{z:|z|=R\}-\bigcup_{n=1}^{\infty} U\left(a_{n}, \frac{\varepsilon a_{n}}{M_{6}\left(\log a_{n}\right)^{\alpha}}\right)
$$

such that $\left|f\left(z_{1}\right)\right|=2$. From (4) we see that $|f(z)| \geqq 1$ in

$$
\left\{z: R_{k} \leqq|z| \leqq R\right\}-\bigcup_{n=1}^{\infty} U\left(a_{n}, M_{6} d_{n}\right) .
$$

We set $g(\zeta)=f\left(R+\zeta^{2}\right)$ and let $h(\zeta)=R+\zeta^{2}$ be the function which maps $\operatorname{Im} \zeta \geqq 0$ onto the $z$-plane. We denote

$$
\begin{aligned}
& A=h^{-1}(\{z:|z|<R\}), \\
& A_{1}=h^{-1}\left(\left\{z:|z| \leqq R_{k}\right\}\right)
\end{aligned}
$$

and $\zeta_{1}=h^{-1}\left(z_{1}\right)$. Then $\zeta_{1}$ lies on the boundary of $A$ and $\pi / 4<\arg \zeta_{1}<3 \pi / 4$. 
If $t<(\sqrt{2}-1) \sqrt{R}$, then $U(i \sqrt{R}, t) \subset A$. Let us suppose that there exists $t$,

$$
(\sqrt{2}-1) \sqrt{R} \leqq t<\sqrt{R}\left(1-\frac{1}{B_{4} \log R}\right),
$$

where $B_{4}=\min \left\{(\log R)^{1 / 8}, \sqrt{K_{k}}\right\}$ such that $|g(\zeta)| \geqq 2$ in $U(i \sqrt{R}, t)-A$ and that there exists $\zeta_{3}$ lying on $|\zeta-i \sqrt{R}|=t$ outside $A$ satisfying $\left|g\left(\zeta_{3}\right)\right|=2$.

We denote $G=U(i \sqrt{R}, t)-A_{1}$, and let $\omega(\zeta)$ be the harmonic measure with respect to $G$ of that part of the boundary of $G$ which is common with $A_{1}$. There exists an absolute constant $B_{3}>0$ such that $U\left(i \sqrt{R}, 4 B_{3} \sqrt{R}\right) \subset A_{1}$. On the boundary of $G$ we have

$$
\omega(\zeta) \geqq \frac{\log (t /|\zeta-i \sqrt{R}|)}{\log \left(t /\left(B_{3} \sqrt{\bar{R}}\right)\right)},
$$

and from the harmonicity we conclude that (8) holds in $G$.

Let $p$ be the greatest integer such that $\gamma_{p} \subset U(0, R)$. For $n \leqq p$, we denote

$$
Q_{n}=\left\{\zeta: R+\zeta^{2} \in \gamma_{n}, \operatorname{Im} \zeta \geqq 0\right\},
$$

and $V_{n}$ is the open domain bounded by $Q_{n}$. If $V_{n} \cap G \neq \emptyset$, we denote by $v_{n}$ the harmonic measure of $Q_{n} \cap G$ with respect to $G$, and

$$
v(\zeta)=\sum_{V_{n} \cap G \neq \varnothing} v_{n}(\zeta)
$$

Let $G_{0}=G-\bigcup_{n=1}^{p} V_{n}$. We note that $v_{n}(\zeta) \leqq \omega_{n}\left(R+\zeta^{2}\right)$ in $G_{0}$ and conclude from (5) that

if $\zeta$ lies in

$$
v(\zeta) \leqq \lambda\left(R+\zeta^{2}\right) \leqq \frac{2 B_{2} \log \log R}{(\log R)^{2}}
$$

$$
G_{1}=G-\left\{\zeta: 0<\operatorname{Im} \zeta<\sqrt{R},|\operatorname{Re} \zeta|<\sqrt{R}(\log R)^{-6}\right\} .
$$

We choose $\zeta_{4}$ by the equation

We may assume that $\zeta_{4} \in G_{1}$,

$$
\zeta_{4}-i \sqrt{R}=\left(\zeta_{3}-i \sqrt{R}\right)\left(1-\frac{1}{B_{4}^{2} \log R}\right) .
$$

It follows from (3) that

$$
\omega\left(\zeta_{4}\right) \geqq \frac{1}{\log \left(1 / B_{3}\right) B_{4}^{2} \log R} .
$$

$$
\log |g(\zeta)| \geqq K_{k}^{2} \log R_{k}(\omega(\zeta)-v(\zeta))
$$

on the boundary of $G_{0}$, and since $\log |g(\zeta)|$ is superharmonic in $G_{0}$, we conclude that (11) holds in $G_{0}$. Therefore we may deduce from (9) and (10) that $\log \left|g\left(\zeta_{4}\right)\right| \geqq \sqrt{K_{k}}$.

Since now $\left|g\left(\zeta_{3}\right)\right| \leqq 2,\left|g\left(\zeta_{4}\right)\right| \geqq 10$ and

$$
\left|\zeta_{3}-\zeta_{4}\right| \leqq \frac{t}{B_{4}^{2} \log R},
$$


we conclude from Lemma 2 that the disc

$$
U\left(\zeta_{3}, \frac{K t}{B_{4}^{2} \log R}\right)
$$

contains at least one zero, 1-point or $w_{3}$-point of $g$. This is not possible, since from (7) and the fact that $\zeta_{3}$ lies outside $A$ it follows that $f$ omits the values 0,1 and $w_{3}$ in

$$
U\left(\zeta_{3}, \frac{t}{2 B_{4} \log R}\right) .
$$

Therefore we conclude now that $|g(\zeta)|>2$ in

This implies that

$$
U\left(i \sqrt{R}, \sqrt{R}\left(1-\frac{1}{B_{4} \log R}\right)\right)-A .
$$

$$
\left|\zeta_{1}\right|<\frac{2 \sqrt{R}}{B_{4} \log R}
$$

because $\zeta_{1}$ lies on the boundary of $A$ and $\left|g\left(\zeta_{1}\right)\right|=2$. If we choose

$$
\zeta_{5}=\frac{8(1+i) \sqrt{R}}{B_{4} \log R},
$$

then $\zeta_{5}$ lies outside $A$, and we see in the same manner as above, $\zeta_{5}$ taking the role of $\zeta_{4}$, that

$$
\log \left|g\left(\zeta_{5}\right)\right| \geqq \sqrt{K_{k}} \text {. }
$$

We set $z_{2}=R+\zeta_{5}^{2}$. Then $\left|f\left(z_{2}\right)\right|>10$ and

$$
\left|z_{1}-z_{2}\right| \leqq\left|\zeta_{1}\right|^{2}+\left|\zeta_{5}\right|^{2} \leqq \frac{500 R}{B_{4}^{2}(\log R)^{2}} .
$$

Using Lemma 2 again, we conclude that the disc

$$
C_{0}=U\left(z_{1}, \frac{500 K R}{B_{4}^{2}(\log R)^{2}}\right)
$$

contains at least one point of $S$. From the choices of $B_{4}$ and $z_{1}$ it follows that if $k$ is large, then $C_{0}$ cannot contain any point of $S$, and we conclude that, if $k$ is large, then $|f(z)| \geqq 2$ in

$$
\left\{z: R_{k} \leqq|z| \leqq R_{k} e^{37}\right\}-\bigcup_{n=1}^{\infty} U\left(a_{n}, \frac{\varepsilon a_{n}}{M_{6}\left(\log a_{n}\right)^{\alpha}}\right),
$$

which implies together with (4) that

$$
|f(z)| \geqq 1
$$

in

$$
\left\{z: R_{k} \leqq|z| \leqq R_{k} e^{37}\right\}-\bigcup_{n=1}^{\infty} U\left(a_{n}, M_{6} d_{n}\right) .
$$


We begin with $t_{1}=R_{k}$, where $k$ is large, and choose $\varrho_{2}, R_{k} e^{36}<\varrho_{2}<R_{k} e^{37}$, such that there are no poles of $f$ on $|z|=\varrho_{2}$ and that

$$
U\left(\varrho_{2}, \frac{\varepsilon \varrho_{2}}{4\left(\log \varrho_{2}\right)^{\alpha}}\right) \cap S=\emptyset .
$$

If $\gamma_{p}$ is a boundary component of

$$
G_{2}=\left\{z: R_{k}<|z|<\varrho_{2}\right\}-\bigcup_{n=1}^{\infty} U\left(a_{n}, M_{6} d_{n}\right)
$$

we denote by $v_{p}$ its harmonic measure with respect to $G_{2}$. Let $u$ be the function harmonic in $R_{k}<|z|<\varrho_{2}$ which has the boundary values $\log |f(z)|$ on $|z|=\varrho_{2}$ and 0 on $|z|=R_{k}$. We denote $\beta_{p}=\max \left\{u(z): z \in \gamma_{p}\right\}$ if $a_{p}$ lies in $R_{k}<|z|<\varrho_{2}$.

On the boundary of $G_{2}$ we have

$$
\log |f(z)| \geqq u(z)-\sum \beta_{p} v_{p}(z),
$$

and from the superharmonicity of $\log |f(z)|$ it follows that (14) holds in $G_{2}$, especially on $|z|=t_{2}$ where $t_{2}, \varrho_{2} / 4<t_{2}<\varrho_{2} / 2$, is chosen such that $\left|t_{2}-a_{n}\right| \geqq \varrho_{2}\left(\log \varrho_{2}\right)^{-3}$ for all $n$.

Let $z$ lie on the circle $|z|=t_{2}$. If $(8 / 9) \varrho_{2} \leqq a_{p}<\varrho_{2}$, then we see from Lemma 6 that

Since

$$
\beta_{p} \leqq 16 m\left(\varrho_{2}, \infty\right) \varepsilon^{-1}\left(\log \varrho_{2}\right)^{\alpha}
$$

$$
v_{p}(\zeta) \leqq \frac{\log \left(2 \varrho_{2} /\left|\zeta-a_{p}\right|\right)}{\log \left(2 \varrho_{2} /\left(M_{6} d_{p}\right)\right)}
$$

in $G_{2}$, we conclude from $(\mathrm{K})$ that

$$
v_{p}(z) \leqq \frac{2 \log 8}{H\left(\log \varrho_{2}\right)^{2+\alpha}} .
$$

The number of the points $a_{p}$ satisfying $(8 / 9) \varrho_{2} \leqq a_{p}<\varrho_{2}$ is at most $\varepsilon^{-1}\left(\log \varrho_{2}\right)^{\alpha}$, and we see that the sum $\sum_{1}$ over these $a_{p}$ satisfies

$$
\sum_{1} \beta_{p} v_{p}(z) \leqq \frac{32 \log 8}{H \varepsilon^{2}} m\left(\varrho_{2}, \infty\right) \leqq \frac{1}{24} m\left(\varrho_{2}, \infty\right)
$$

If $R_{k}<a_{p}<(8 / 9) \varrho_{2}$, then it follows from Lemma 6 that $\beta_{p}=O\left(m\left(\varrho_{2}, \infty\right)\right)$, and we conclude from (5) that the sum $\sum_{2}$ over these $a_{p}$ satisfies

$$
\sum_{2} \beta_{p} v_{p}(z) \leqq O\left(m\left(\varrho_{2}, \infty\right) \lambda(z)\right)=o\left(m\left(\varrho_{2}, \infty\right)\right) \leqq \frac{1}{24} m\left(\varrho_{2}, \infty\right)
$$

if $k$ was chosen sufficiently large. Combining these estimates with (14), we deduce that

$$
\log |f(z)| \geqq u(z)-\frac{1}{12} m\left(\varrho_{2}, \infty\right)
$$


on $|z|=t_{2}$, and from Lemma 6 we see now that

$$
\log |f(z)| \geqq \frac{1}{12} m\left(\varrho_{2}, \infty\right)
$$

for all $z$ lying on $|z|=t_{2}$.

Since

$$
\lim _{r \rightarrow \infty} \frac{m(r, \infty)}{\log r}=\infty,
$$

taking $t_{2}$ instead of $R_{k}$, we get $|f(z)| \geqq 1$ for

$$
z \in\left\{z: t_{2} \leqq|z| \leqq t_{2} e^{37}\right\}-\bigcup_{n=1}^{\infty} U\left(a_{n}, M_{6} d_{n}\right),
$$

and continuing this process inductively, we conclude that there exists $R_{0}>0$ such that $|f(z)| \geqq 1$ for all $z$ lying in

$$
\left\{z:|z|>R_{0}\right\}-\bigcup_{n=1}^{\infty} U\left(a_{n}, M_{6} d_{n}\right)
$$

We choose a sequence $r_{n}, r_{1}>\left(4+R_{0}\right)^{100}$, such that $r_{n-1}^{2}<r_{n}<2 r_{n-1}^{2}$, there exist no poles of $f$ on $|z|=r_{n}$, and

$$
U\left(r_{n}, \frac{\varepsilon r_{n}}{4\left(\log r_{n}\right)^{\alpha}}\right) \cap S=\emptyset .
$$

The sequence $x_{n}$ is chosen such that $r_{n}^{1 / 100} / 2<x_{n} \leqq r_{n}^{1 / 100}$ and that (16) is satisfied if $r_{n}$ is replaced by $x_{n}$.

Let $u$ be the function harmonic in $x_{n}<|z|<r_{n}$ which has the boundary values $\log |f(z)|$ on $|z|=r_{n}$ and 0 on $|z|=x_{n}$. For those $a_{p}$ which lie in $x_{n}<|z|<r_{n}$, we set

$$
w_{p}(z)=\frac{\log \left(2 r_{n} /\left|z-a_{p}\right|\right)}{\left.\log \left(2 r_{n} / M_{6} d_{p}\right)\right)} .
$$

Using Lemma 6 as in the proof of Theorem 10, we deduce that

on $|z|=r_{n-1}$.

$$
\log |f(z)| \geqq u(z)-2 m\left(r_{n}, \infty\right) \sum \frac{r_{n}+a_{p}}{r_{n}-a_{p}} w_{p}(z)
$$

Let $|z|=r_{n-1}$. If $r_{n} / 2 \leqq a_{p}<r_{n}$, then

$$
\frac{r_{n}+a_{p}}{r_{n}-a_{p}} w_{p}(z) \leqq \frac{32\left(\log r_{n}\right)^{\alpha} \log 8}{H \varepsilon\left(\log r_{n}\right)^{2+\alpha}},
$$

the number of these $a_{p}$ is at most $2 \varepsilon^{-1}\left(\log r_{n}\right)^{\alpha}$, and the sum $\sum_{1}$ over these $a_{p}$ satisfies

$$
\sum_{1} \frac{r_{n}+a_{p}}{r_{n}-a_{p}} w_{p}(z) \leqq \frac{64 \log 8}{H \varepsilon^{2}} \leqq \frac{1}{100} .
$$


If $x_{n}<a_{p}<r_{n} / 2$, then

$$
\frac{r_{n}+a_{p}}{r_{n}-a_{p}} w_{p}(z) \leqq \frac{6 \log r_{n}}{H\left(100^{-1} \log r_{n}\right)^{2+\alpha}} .
$$

Let $n(r)$ be the counting function of the sequence $a_{n}$. From $(\mathrm{J})$ we get

which implies that

$$
n\left(e^{k}\right)-n\left(e^{k-1}\right) \leqq \frac{8}{\varepsilon} k^{\alpha},
$$

$$
n\left(e^{k}\right) \leqq \frac{8}{\varepsilon} \sum_{s=1}^{k} s^{\alpha}+O(1)=\left(\frac{8}{\varepsilon}+o(1)\right) \int_{i}^{k} x^{\alpha} d x .
$$

From this it follows that $n(r) \leqq 8 \varepsilon^{-1}(\log r)^{1+\alpha}$ for all large $r$. Therefore the number of $a_{p}$ satisfying $x_{n}<a_{p}<r_{n} / 2$ is at most $8 \varepsilon^{-1}\left(\log r_{n}\right)^{1+\alpha}$, and we deduce from (19) that the sum $\sum_{2}$ over these $a_{p}$ satisfies

$$
\sum_{2} \frac{r_{n}+a_{p}}{r_{n}-a_{p}} w_{p}(z) \leqq \frac{48(100)^{2+\alpha}}{H \varepsilon} \leqq \frac{1}{100} .
$$

Combining the estimates (18) and (20) with (17) we conclude that

$$
\log |f(z)| \geqq u(z)-\frac{1}{25} m\left(r_{n}, \infty\right)
$$

on $|z|=r_{n-1}$.

From Lemma 6 we get

$$
u(z) \geqq \frac{98}{198}(1+o(1)) m\left(r_{n}, \infty\right),
$$

and this implies together with (21) that

$$
m\left(r_{n-1}, \infty\right) \geqq \frac{9}{20} m\left(r_{n}, \infty\right)
$$

In the same manner as in the proof of Theorem 10, we see that (22) implies that

$$
T(r, f)=O\left((\log r)^{3 / 2}\right),
$$

and that this leads to the impossibility that $\delta(0, f) \geqq \delta(\infty, f)$. Theorem 11 is proved.

\section{References}

[1] Anderson, J. M., and J. Clunie: Picard sets of entire and meromorphic functions. - Ann. Acad. Sci. Fenn. Ser. A I 5, 1980, 27-43.

[2] BAKer, I. N.: Linear Picard sets for entire functions. - Math. Nachr. 64, 1974, 263-276.

[3] BAKer, I. N., and L. S. O. Liverpool: Picard sets for entire functions. - Math. Z. 126, 1972, $230-238$.

[4] Baker, I. N., and L. S. O. Liverpool: Further results on Picard sets of entire functions. - Proc. London Math. Soc. (3) 26, 1973, 82-98. 
[5] Hayman, W. K.: Meromorphic functions. - Clarendon Press, Oxford, 1964.

[6] Hayman, W. K.: Slowly growing integral and subharmonic functions. - Comment. Math. Helv. 34, 1960, 75-84.

[7] LeHto, O.: A generalization of Picard's theorem. - Ark. Mat. 3, 1958, 495-500.

[8] Matsumoto, K.: Remark to Lehto's paper “A generalization of Picard's theorem”. - Proc. Japan. Acad. 38, 1962, 636-640.

[9] Matsumoto, K.: Some remarks on Picard sets. - Ann. Acad. Sci. Fenn. Ser. A I 403, 1967, $1-17$.

[10] Toppila, S.: Picard sets for meromorphic functions. - Ann. Acad. Sci. Fenn. Ser. A I 417, $1967,1-24$.

[11] ToppIla, S.: Some remarks on the value distribution of meromorphic functions. - Ark. Mat. 9, 1971, 1-9.

[12] Toppila, S.: Some remarks on linear Picard sets. - Ann. Acad. Sci. Fenn. Ser. A I 569, 1973, $1-17$.

[13] ToppIla, S.: On the value distribution of integral functions. - Ann. Acad. Sci. Fenn. Ser. A I 574, 1974, 1-20.

[14] Toppila, S.: Linear Picard sets for entire functions. - Ann. Acad. Sci. Fenn. Ser. A I 1, 1975, 111-123.

[15] Toppila, S.: On the value distribution of meromorphic functions with adeficient value. - Ann. Acad. Sci. Fenn. Ser. A I 5, 1980, 179-184.

[16] VAliron, G.: Sur les valeurs déficientes des fonctions algebroïdes méromorphes d'ordre nul. - J. Analyse Math. I, 1951, 28-42.

[17] WinkleR, J.: Über Picardmengen ganzer unđ meromorpher Funktionen. - Math. Z. 109, 1969, 191-204.

[18] Winkler, J.: Über Picardmengen ganzer Funktionen. - Manuscripta Math. 1, 1969, 191-199.

[19] WinkLeR, J.: Bericht über Picardmengen ganzer Funktionen. - Topics in analysis, Colloquium on mathematical analysis, Jyväskylä 1970, edited by O. Lehto, I. S. Louhivaara and R. Nevanlinna, Springer-Verlag, Berlin-Heidelberg-New York, 1974, 384-392.

[20] WinkLeR, J.: Ein Kriterium für Picardmengen ganzer Funktionen. - Math. Nachr. 49, 1971, $267-275$.

[21] WinkLeR, J.: Eine Bemerkung über Picardmengen ganzer Funktionen. - Math. Nachr. 52, 1972, 207-216.

[22] WinkLeR, J.: Zur Existenz ganzer Funktionen bei vorgegebener Menge der Nullstellen und Einsstellen. - Math. Z. 168, 1979, 77-85.

University of Helsinki

Department of Mathematics

SF-00100 Helsinki 10

Finland

Received 14 January 1980 